\title{
Convexity properties of superpositions of degenerate bipartite eigenstates
}

\author{
Natalia Giovenale, ${ }^{*}$ Federico M. Pont ${ }^{\dagger}$ Pablo Serra, ${ }^{\ddagger}$ and Omar Osenda ${ }^{\S}$ \\ Facultad de Matemática, Astronomía, Física y Computación, Universidad Nacional de Córdoba y Instituto de Física Enrique Gaviola, \\ CONICET, Avenida Medina Allende s/n, Ciudad Universitaria, CP:X5000HUA Córdoba, Argentina
}

(Received 2 November 2018; published 24 May 2019)

\begin{abstract}
The entanglement content of superpositions of pairs of degenerate eigenstates of a bipartite system is considered in the case that both eigenstates are also eigenstates of the $z$ component of the total angular momentum. It is shown that the von Neumann entropy of the state that is obtained tracing out one of the parts of the system has a definite convexity (concavity) as a function of the superposition parameter and that its convexity (concavity) can be predicted using a quantity of information that measures the entropy shared by the states at the extremes of the superposition. Several examples of two-particle systems, whose eigenfunctions and density matrices can be obtained exactly, are analyzed thoroughly.
\end{abstract}

DOI: 10.1103/PhysRevA.99.052340

\section{INTRODUCTION}

As the old recipe goes, the only thing that someone needs to prepare a pure state with well-defined quantum numbers is a complete set of compatible observables of the quantum system the states of which are to be prepared. Then, applying a given sequence of one-dimensional projectors to an arbitrary pure state, it is possible to label the resulting state with the quantum numbers associated to each projector. The sequence contains only one projector for each observable which is taken from the corresponding spectral decomposition. Only if the observable set is complete the state at the end of the sequence will be an element of the basis that expands the Hilbert space of the system and, consequently, cannot be written as a superposition of states generated following the recipe but with different projectors. Of course, one-dimensional projectors are elusive objects to be actually constructed, in particular when the observable has a continuum spectrum.

The drive to process quantum information, to control the states where it is stored, and to palliate the unwanted effects of decoherence mechanisms has resulted in a host of methods to produce pure or almost pure states in a reliable and repetitive way in different quantum systems. A short list of examples includes effective pure states in magnetic nuclear resonance [1-3], state preparation of coupled electron states in quantum dots [4], and synthesis of arbitrary states in superconducting qubits [5]. Moreover, the availability of quantum states made of specific superpositions of pure states or measurementprepared states could improve the means to perform a given quantum information task [6-9].

Some years ago, Vaziri, Weihs, and Zeilinger [10] showed that for a particular quantum information task, quantum cryptography, the availability of photon states made of

\footnotetext{
*ngiovenale@famaf.unc.edu.ar

†pont@famaf.unc.edu.ar

ॠserra@famaf.unc.edu.ar

§osenda@famaf.unc.edu.ar
}

superpositions of orbital angular momentum eigenstates resulted in an expanded alphabet to be used to code information, with possibilities beyond the simpler alphabet formed by the two polarization states. In Ref. [10] superpositions between Gaussian and Laguerre-Gaussian (LG) states were considered, but over the years numerous other examples of states that are composed of different orbital angular momentum states have been analyzed including photon pairs with entangled orbital angular momentum [11], composite Laguerre-Gaussian beams with tunable intensity and phase distribution [12], and elliptic Gaussian optical vortices [13]. Also, it has been shown experimentally that photonic states with large orbital angular momentum, often called qudits, can be cycled among them using standard optical elements [14].

In other physical systems, to our knowledge, there is neither the requirement of a specific superposition of orbital angular momentum states to perform a task nor a protocol to achieve it, as there is for the neat example for photons presented above. At any rate, this scenario is changing because of the appearance of experiments and theoretical proposals in which the properties of a photonic state are transferred to a condensate system [15].

Nevertheless, there are at least two reasons to consider superpositions of degenerate states. One comes from the properties of many-body eigenstates, and another one arises from considerations about the Hilbert space expanded by all the superpositions that can be formed using a set of pure states. When considering many-body models the eigenstates of the Hamiltonian are, most commonly, degenerate, and depending on the problem that is under study the entanglement content could be calculated for a mixture or for a superposition of them. This subtle point was early acknowledged by Osborne and Nielsen [16]. They studied the behavior of the entanglement in the quantum phase transition that appears in the transverse Ising model and discussed the differences in entanglement content between the low-temperature limit of the Gibbs state, that is, an equally weighted mixture of the two degenerate ground states that the system admits, and the entanglement of one of those ground states. 
Since the work of Osborne and Nielsen [16], the entanglement content of the degenerate eigenstates of many-body problems has been addressed to assess the relationship between long-range interactions and the entanglement of spin pairs separated at different lengths [17], the relationship between entanglement and symmetry in permutation-symmetric states [18], the difference between symmetrical superpositions of ground states and symmetry-breaking ones using mutual information [19], and the entanglement properties of the whole set of eigenstates of different Hamiltonians [20,21]. For instance, in Ref. [17] the entanglement was calculated for equally weighted mixtures of degenerate eigenstates, while Markham in Ref. [18] considered superpositions of states where their coefficients are given by complex numbers with modulus equal to 1 , so the normalization constant of the superposition is equal to the number of states that enters into it.

On the other hand, when all the superpositions of a set of $N$ eigenstates are considered, since they are contained in a compact space, a given continuous functional of the states should reach a set of extrema. So, any given entanglement measure will reach a number of extrema. But, for which superpositions are those extrema achieved? Moreover, is the entanglement measure of superpositions of states convex or concave as a function of the superposition parameter? Also, can these convexity properties be predicted from the knowledge of the states that are being superposed?

Some of us started to contemplate these questions after working with the Calogero model [22]. The Calogero model has many well-documented properties and applications [23], but the one that caught our attention and was the reason to start this paper is the following: the two-particle two-dimensional case has a degenerate ground state if the total wave function considered is antisymmetric under particle permutation. Most commonly, the eigenfunctions are chosen as eigenfunctions of the angular momentum operator component perpendicular to the two-dimensional plane the system inhabits; let us call them $\psi_{ \pm}$. Other usual choices for the basis eigenfunctions are the combinations $\left(\psi_{+} \pm \psi_{-}\right) / \sqrt{2}$. Denoting by $S(\psi)$ the von Neumann entropy of the reduced density operator obtained tracing out one of the particles from the two-particle density operator, $\rho=|\psi\rangle\langle\psi|$, we obtained [22] that $S(\xi) \leqslant S\left(\psi_{+}\right)=$ $S\left(\psi_{-}\right)$for all $\xi=\sqrt{\alpha} \psi_{+}+\sqrt{1-\alpha} \psi_{-}$, where $0 \leqslant \alpha \leqslant 1$. This finding led us to analyze more general examples.

Suppose that $L_{z}$ commutes with $H$, where $H$ is the Hamiltonian with degenerate eigenstates labeled as $\left|\psi_{i}\right\rangle$, and $L_{z}\left|\psi_{i}\right\rangle=m_{i}\left|\psi_{i}\right\rangle$, where $m_{i}$ are some eigenvalues. Consider a superposition $\chi=\sum_{i} \sqrt{\alpha_{i}} \psi_{i}$, such that $S(\chi)$ is convex or concave as a function of the parameters $\alpha_{i}$, which satisfy that $\sum_{i} \alpha_{i}=1$ and $0 \leqslant \alpha_{i} \leqslant 1$. In this paper we study only superpositions of degenerate states with eigenvalues of the form $\pm m \hbar$. We give a criterion that predicts the convexity or concavity of the entropy of the superposition. To avoid the uncertainty and difficulties that arise when numerical solutions are required, we consider a two-particle exactly solvable model, two-interacting harmonic oscillators; a quasiexactly solvable one, the spherium [24,25]; a oneparticle exactly solvable model, the Laguerre-Gaussian onephoton wave function [26]; and the sum of two angular momentum operators. This set of examples has been chosen because the one-party reduced density matrix can be exactly calculated and its eigenvalues can be obtained to arbitrary precision. For bipartite two-particle models one particle must be traced out from the whole two-particle density matrix, while for the one-particle model what is traced out is one of the two relevant spatial degrees of freedom. This procedure has been used to assess the separability of a given wave function as a product of two functions that depend on separate variables [27] and has been also considered to assess the entanglement between degrees of freedom of one-particle systems, for instance, polarization and spatial degrees of freedom in one-photon states [28] or Rydberg-like harmonic states [29].

The paper is organized as follows. In Sec. II a criterion to predict the convexity properties of the entropy of superposition of eigenstates is presented. In Secs. III, IV, V, and VI we present, respectively, the calculation of the eigenstates; entropies and the criterion for two interacting oscillators; two electrons confined in the surface of a sphere (the spherium); and one-photon Laguerre-Gaussian states and the addition of two angular momentum operators. We defer a number of mathematical details to the Appendices. Finally in Sec. VII we discuss our results and perspectives of the research.

\section{THE CRITERION}

Consider a bipartite composite system with Hilbert space $\mathcal{H}=\mathcal{H}_{A} \otimes \mathcal{H}_{B}$, where $\mathcal{H}_{A}$ and $\mathcal{H}_{B}$ are the Hilbert spaces of the two subsystems $A$ and $B$, the dimensions of which are equal, $\operatorname{dim}\left(\mathcal{H}_{A}\right)=\operatorname{dim}\left(\mathcal{H}_{B}\right)$.

For a given pure state in $\mathcal{H},|\psi\rangle$, the reduced density matrices $\rho_{A}$ and $\rho_{B}$ are given by

$$
\rho_{A}=\operatorname{Tr}_{B}(|\psi\rangle\langle\psi|), \quad \rho_{B}=\operatorname{Tr}_{A}(|\psi\rangle\langle\psi|) .
$$

Both reduced density matrices are isospectral and have associated eigenvalue problems, i.e.,

$$
\rho_{A} \varphi_{i}^{A}=\lambda_{i}^{A} \varphi_{i}^{A},
$$

and

$$
\rho_{B} \varphi_{i}^{B}=\lambda_{i}^{B} \varphi_{i}^{B},
$$

where $\lambda_{i}^{A}\left(\lambda_{i}^{B}\right)$ are the eigenvalues and $\varphi_{i}^{A}\left(\varphi_{i}^{B}\right)$ are the eigenvectors of $\rho_{A}\left(\rho_{B}\right)$. It is convenient to introduce two other Hilbert spaces, $V_{A}=\operatorname{span}\left(\left\{\varphi_{j}^{A}\right\}\right)$, where the only eigenvectors $\varphi_{j}^{A}$ that enter into the span are those such that its corresponding eigenvalues satisfy $\lambda_{j}^{A}>0$, and equivalently for $V_{B}$.

The considerations over the Hilbert spaces defined above become clear when computing the quantum relative entropy, a quantity commonly used to compare two quantum states, $\rho$ and $\sigma$. The quantum relative entropy of $\rho$ with respect to $\sigma$ is given by [30]

$$
S(\rho \| \sigma)=-\operatorname{Tr}(\rho \log \sigma)-S(\rho)=\operatorname{Tr} \rho(\log \rho-\log \sigma),
$$

where $S(\rho)$ is the von Neumann entropy:

$$
S(\rho)=-\operatorname{Tr} \rho \log \rho .
$$

If $\operatorname{supp}(\rho) \bigcap \operatorname{ker}(\sigma) \neq 0$ then $S(\rho \| \sigma)=\infty$, where $\operatorname{supp}(\rho)$ and $\operatorname{ker}(\sigma)$ stand for the support and kernel of the $\rho$ and $\sigma$ states, respectively. The divergence and other properties of 
the relative entropy can be analyzed more directly using the spectral decompositions

$$
\rho=\sum_{i} p_{i}\left|v_{i}\right\rangle\left\langle v_{i}\left|, \quad \sigma=\sum_{i} q_{i}\right| w_{i}\right\rangle\left\langle w_{i}\right|,
$$

in terms of which the quantum relative entropy can be calculated as

$$
S(\rho \| \sigma)=\sum_{i} p_{i}\left(\log p_{i}-\sum_{j}\left(\log q_{j}\right) P_{i j}\right),
$$

where $P_{i j}=\left|\left\langle v_{i} \mid w_{j}\right\rangle\right|^{2}$.

Some remarks are in order. If the states $\rho$ and $\sigma$ have degenerate eigenvalues, each one of the decompositions in Eq. (6) is not unique, since the corresponding eigenvectors $\left|v_{i}\right\rangle$ and $\left|w_{i}\right\rangle$ can be chosen in different ways. This possibility leads to the undesirable result that the relative entropy could depend on a particular election of the eigenvectors that corresponds to a degenerate eigenvalue. Since we intend to introduce an informationlike quantity that allows us to compare the reduced density matrices of different superpositions of Hamiltonian eigenstates, the quantity to be defined must be calculable even when the two drawbacks mentioned above are present.

If $H$ is the Hamiltonian of the composite system, we will focus on some particular superpositions of degenerate bound states which are also eigenstates of the $z$ component of the total angular momentum $L_{z}$. In particular, if $\left|\psi_{0}\right\rangle$ and $\left|\psi_{1}\right\rangle$ are two such eigenstates, we will consider the superposition

$$
\left|\psi_{\alpha}\right\rangle=\sqrt{\alpha}\left|\psi_{0}\right\rangle+\sqrt{1-\alpha}\left|\psi_{1}\right\rangle, \quad 0 \leqslant \alpha \leqslant 1 .
$$

Let us define the not-shareable entropy of $\rho_{A, 0}$ with respect to $\rho_{A, 1}$ as

$$
S_{\mathrm{ns}}\left(\rho_{A, 0}\right)=-\sum_{\lambda_{i}^{A, 0}>0} \Theta\left[\lambda_{i}^{A, 0}-\left\langle\rho_{A, 1}\right\rangle_{i}\right] \log _{2}\left(\lambda_{i}^{A, 0}\right),
$$

where

$$
\rho_{A, \alpha}=\operatorname{Tr}_{B}\left(\left|\psi_{\alpha}\right\rangle\left\langle\psi_{\alpha}\right|\right),
$$

$\left\langle\rho_{A, 1}\right\rangle_{i}=\operatorname{Tr}\left(P_{i}^{A, 0} \rho_{A, 1}\right), P_{i}^{A, 0}$ is a one-dimensional projector associated to $\lambda_{i}^{A, 0}$, and $\Theta[x]=x \theta(x)$, where $\theta(x)$ is the Heaviside step function. It is clear that when the eigenvalues of $\rho_{A, 0}$ are degenerate the projectors $P_{i}^{A, 0}$ are not uniquely defined. So, decomposing the sum in Eq. (9) in terms of the degenerate and nondegenerate eigenvalues we obtain

$$
S_{\mathrm{ns}}\left(\rho_{A, 0}\right)=-\sum_{i} \sum_{\nu=1}^{\operatorname{deg}\left(\lambda_{i}^{A, 0}\right)} \Theta\left[\lambda_{i}^{A, 0}-\left\langle\rho_{A, 1}\right\rangle_{i, v}\right] \log _{2}\left(\lambda_{i}^{A, 0}\right),
$$

where the first sum runs over the different eigenvalues and the second one runs over the degeneracy of the corresponding eigenvalue. With the previous definitions, we now define the entropy on which we will base our paper. The not-shared entropy of $\rho_{A, 0}$ with respect to $\rho_{A, 1}$ is given by

$S_{\mathrm{NS}}\left(\rho_{A, 0}\right)=-\sum_{i} \min \left\{\sum_{\nu=1}^{\operatorname{deg}\left(\lambda_{i}^{A, 0}\right)} \Theta\left[\lambda_{i}^{A, 0}-\left\langle\rho_{A, 1}\right\rangle_{i, \nu}\right]\right\} \log _{2}\left(\lambda_{i}^{A, 0}\right)$, where the minimum must be obtained in each degenerate subspace associated to a degenerate eigenvalue of $\rho_{A, 0}$. The minimum can be obtained analytically for low degeneracy proceeding as follows. To simplify the notation let us call $\lambda$ the degenerate eigenvalue of interest and $\lambda_{1}$ and $\lambda_{2}$ the eigenvalues of $\rho_{A, 1}$, and it is clear that we are assuming a twofold degeneracy. In the corresponding subspace, $\left\langle\rho_{A, 1}\right\rangle_{1}+$ $\left\langle\rho_{A, 1}\right\rangle_{2}=\lambda_{1}+\lambda_{2}$, so

$$
\tilde{S}=\min \left\{\Theta\left[\lambda-\left\langle\rho_{A, 1}\right\rangle_{1}\right]+\Theta\left[\lambda-\left\langle\rho_{A, 1}\right\rangle_{2}\right]\right\} \log _{2}(1 / \lambda)
$$

can be calculated explicitly as

$$
\tilde{S}=\left\{\begin{array}{ll}
{\left[2 \lambda-\left(\lambda_{1}+\lambda_{2}\right)\right] \log _{2}(1 / \lambda)} & \text { if } \lambda>\frac{\lambda_{1}+\lambda_{2}}{2} \\
0 & \text { otherwise }
\end{array} .\right.
$$

Once the not-shared entropy is defined we introduce, in a similar fashion, the remaining entropy of state $\rho_{A, 0}$, which is given by

$$
S_{R}\left(\rho_{A, 0}\right)=S\left(\rho_{A, 0}\right)-S_{\mathrm{NS}}\left(\rho_{A, 0}\right) .
$$

We are now in conditions to state the criterion about the convexity properties of the entropy of a superposition of two degenerate states, like the one defined in Eq. (8). If both states, $\psi_{0}$ and $\psi_{1}$, are eigenfunctions of the $z$ component of the total angular momentum operator, $L_{z}$, with eigenvalues $\pm m \hbar$, then

$$
\begin{aligned}
& S\left(\rho_{A, \alpha}\right) \leqslant \alpha S\left(\rho_{A, 0}\right)+(1-\alpha) S\left(\rho_{A, 1}\right) \\
& \text { if } \quad S_{\mathrm{NS}}\left(\rho_{A, 0}\right)<S_{R}\left(\rho_{A, 0}\right)
\end{aligned}
$$

and

$$
\begin{aligned}
& S\left(\rho_{A, \alpha}\right) \geqslant \alpha S\left(\rho_{A, 0}\right)+(1-\alpha) S\left(\rho_{A, 1}\right) \\
& \text { if } \quad S_{\mathrm{NS}}\left(\rho_{A, 0}\right)>S_{R}\left(\rho_{A, 0}\right) .
\end{aligned}
$$

The criterion predicts exactly the convexity properties expected for the entropy, and this can be noted by the quantity

$$
Q_{c}=\operatorname{sgn}\left[S_{R}\left(\rho_{A, 0}\right)-S_{\mathrm{NS}}\left(\rho_{A, 0}\right)\right],
$$

which has the value +1 when the entropy $S\left(\rho_{A, \alpha}\right)$ is convex and -1 when it is concave.

Then, the criterion establishes a relationship between the convexity of the entropy of a superposition state with an entropic quantity that depends only on the extremal states of such superposition. In the following sections we will test the criterion in several systems and show how it correctly predicts how state superposition can lead to more or less information entropy.

It is worth noting that the criterion can be stated also in terms of the state $\rho_{A, 1}$ with respect to the state $\rho_{A, 0}$. Even more, it seems desirable to state a criterion in which both states play more symmetrical roles. For the moment we prefer to use the criterion as stated from Eqs. (9)-(18). The criterion compares a given state, say $\rho_{A, 0}$, with another, say $\rho_{A, 1}$, so the not-shared entropy contains information of $\rho_{A, 0}$ that cannot be obtained from state $\rho_{A, 1}$ measuring the last in the basis of eigenprojectors of the former. If $V_{A} \cap V_{B}=\phi$ then $S_{\mathrm{NS}}\left(\rho_{A, 0}\right)=S\left(\rho_{A, 0}\right)$. In this sense, the not-shared entropy measures how different are the states when both are measured using the projectors associated to one of them. 
The remaining entropy quantifies how much information can be obtained about $\rho_{A, 0}$ when $\rho_{A, 1}$ is measured using the projectors associated to $\rho_{A, 0}$. This can be seen rather directly when both states, $\rho_{A, 0}$ and $\rho_{A, 1}$, have nondegenerate eigenvalues and the same set of eigenvectors. In that case,

$$
S_{R}=-\sum_{i} \min \left(\lambda_{i}^{A, 0}, \lambda_{i}^{A, 1}\right) \log _{2}\left(\lambda_{i}^{A, 0}\right),
$$

and the sum runs over the eigenvalues $\lambda_{i}^{A, 0}$ the eigenvectors of which belong to $V_{A} \bigcap V_{B}$.

The not-shared entropy, as proposed in Eq. (12), depends on the spectral decomposition of both reduced density operators $\rho_{A, 0}$ and $\rho_{A, 1}$. This dependency does not imply a restriction from the point of view of the calculations in concrete systems but, as is the case for entanglement measures, a definition in terms of a minimization over a Hilbert space can offer a more general point of view or an operational procedure to determine the not-shared entropy. We will return to this subject once the example considering states in finite-dimensional Hilbert spaces, Sec. VI, has been analyzed thoroughly.

In the next sections we apply the criterion to several bipartite systems. The different Hamiltonians allow us to carry the calculations to obtain the reduced density matrices analytically.

\section{TWO INTERACTING TWO-DIMENSIONAL OSCILLATORS}

A well-known exactly solvable problem consists of two particles interacting harmonically confined in an harmonic trap. We use units such that $\hbar=1, m=1, \omega=1$, where $m$ is the mass and $\omega$ is the frequency of both oscillators. The Hamiltonian is given by

$$
H\left(\vec{x}_{1}, \vec{x}_{2}\right)=-\frac{1}{2} \nabla_{1}^{2}-\frac{1}{2} \nabla_{2}^{2}+\frac{1}{2}\left|\vec{x}_{1}\right|^{2}+\frac{1}{2}\left|\vec{x}_{2}\right|^{2}+\lambda\left|\vec{x}_{1}-\vec{x}_{2}\right|^{2},
$$

where $\vec{x}_{i}=\left(x_{i}, y_{i}\right), \nabla_{i}^{2}$ is the two-dimensional Laplacian, and $i=1,2$ is the index numbering the particles.

The parameter $\lambda$ allows us to switch from a noninteracting system, $\lambda=0$, to an interacting one, $\lambda>0$. The symmetries and quantum numbers of the wave functions can be chosen at convenience. Since the superpositions in which we are interested are composed of eigenfunctions with degenerate energy eigenvalues, but with different angular momentum eigenvalues, it is useful to derive expressions for the wave functions in coordinates where the system can be recast as a noninteracting one. So, introducing the centered and relative coordinates

$$
\vec{R}=\vec{x}_{1}+\vec{x}_{2}, \quad \vec{r}=\vec{x}_{1}-\vec{x}_{2},
$$

respectively, the Hamiltonian in Eq. (20) can be written as

$$
\begin{aligned}
H(\vec{R}, \vec{r}) & =H_{\omega_{R}}(\vec{R})+H_{\omega_{r}}(\vec{r}) \\
& =-\nabla_{R}^{2}+\frac{1}{4}|\vec{R}|^{2}-\nabla_{r}^{2}+\left(\frac{1}{4}+\lambda\right)|\vec{r}|^{2},
\end{aligned}
$$

where $H_{\omega}$ is a two-dimensional harmonic oscillator Hamiltonian, with frequency $\omega$ and mass equal to $1 / 2$. The oscillator that depends on the relative coordinates has $\omega_{r}=\sqrt{4 \lambda+1}$, while the other, that depends on the centered coordinates, has
$\omega_{R}=1$. The exact eigenfunctions and eigenvalues of the oneparticle Hamiltonians in Eq. (22) are well known in several coordinate systems [31]. The two-particle eigenfunctions can now be written as a product of a pair of one-particle ones.

The one-particle cylindrical eigenvectors, $|m, n\rangle$, satisfy

$$
\begin{aligned}
H_{\omega}|n, m\rangle & =(2 n+|m|+1) \omega|n, m\rangle, \\
L_{z}^{(1)}|n, m\rangle & =m|n, m\rangle,
\end{aligned}
$$

where $\omega$ is either $\omega_{R}$ or $\omega_{r}$, and $L_{z}^{(1)}$ is the $z$ component of the one-particle angular momentum.

Collecting the results given above, the two-particle eigenvector, $|n, m, l, p\rangle=|n, m\rangle|l, p\rangle$, satisfies

$$
\begin{aligned}
& H|n, m, l, p\rangle \\
& \quad=\left[(2 n+|m|+1) \omega_{R}+(2 l+|p|+1) \omega_{r}\right]|n, m, l, p\rangle
\end{aligned}
$$

and

$$
L_{z}|n, m, l, p\rangle=(m+p)|n, m, l, p\rangle,
$$

where $L_{z}=L_{z_{R}}+L_{z_{r}}$.

Equations (24) and (25) show that, when $\sqrt{4 \lambda+1}$ is a natural number, there are many different ways to combine the quantum numbers $n, m, l$, and $p$ to obtain degenerate eigenfunctions with different values of angular momentum quantum number.

The traces in Eq. (1) over $A$ and $B$ have the meaning of particle 1 and 2 . Hence the reduced density matrix of a particular angular momentum state is

$$
\rho_{A}\left(\vec{x}_{1}, \vec{x}_{1}^{\prime}\right)=\int d^{2} x_{2} \psi_{n, m, l, p}^{*}\left(\vec{x}_{1}, \vec{x}_{2}\right) \psi_{n, m, l, p}\left(\vec{x}_{1}^{\prime}, \vec{x}_{2}\right)
$$

The states $\left|\psi_{0}\right\rangle$ and $\left|\psi_{1}\right\rangle$ are now identified with two different angular momentum states $|n, m, l, p\rangle$ that have the same energy and opposite eigenvalues for $L_{z}$. The reduced density matrix for the mixed state $\rho_{A, \alpha}$ is defined analogously to $\rho_{A}\left(\vec{x}_{1}, \vec{x}_{1}^{\prime}\right)$ in Eq. (26). We explain how to obtain an exact expression for $\rho_{A}\left(\vec{x}_{1}, \vec{x}_{1}^{\prime}\right)$ and how to compute its eigenvalues in Appendix B.

\section{A. Noninteracting harmonic oscillators, $\lambda=0$}

The bipartite states are labeled with the four quantum numbers $n, m, l$, and $p$. In this section we will consider superpositions of states that satisfy $|m+p|=1$ or 2 ; there is no $a$ priori restriction to the values of the quantum numbers $n$ and $l$ but the one imposed by Eq. (24). However, since we use a finite basis from which we obtain the eigenvalues of $\rho_{A, \alpha}$, we consider the states for $n, l \leqslant 3$.

Figure 1 shows the behavior of the von Neumann entropy as a function of the superposition parameter $\alpha$. Both possible curvatures, or convexities, can be clearly appreciable according to the states that enter into the superposition. In Table I the values of von Neumann, remaining, and not-shared entropies and $Q_{c}$ are listed for all the cases shown in Fig. 1. The criterion predicts correctly what convexity is to be expected.

\section{B. Interacting harmonic oscillators, $\lambda \neq 0$}

Despite the fact that a two-particle system, both confined by a harmonic potential and interacting harmonically, is exactly solvable, the evaluation of the necessary matrix 

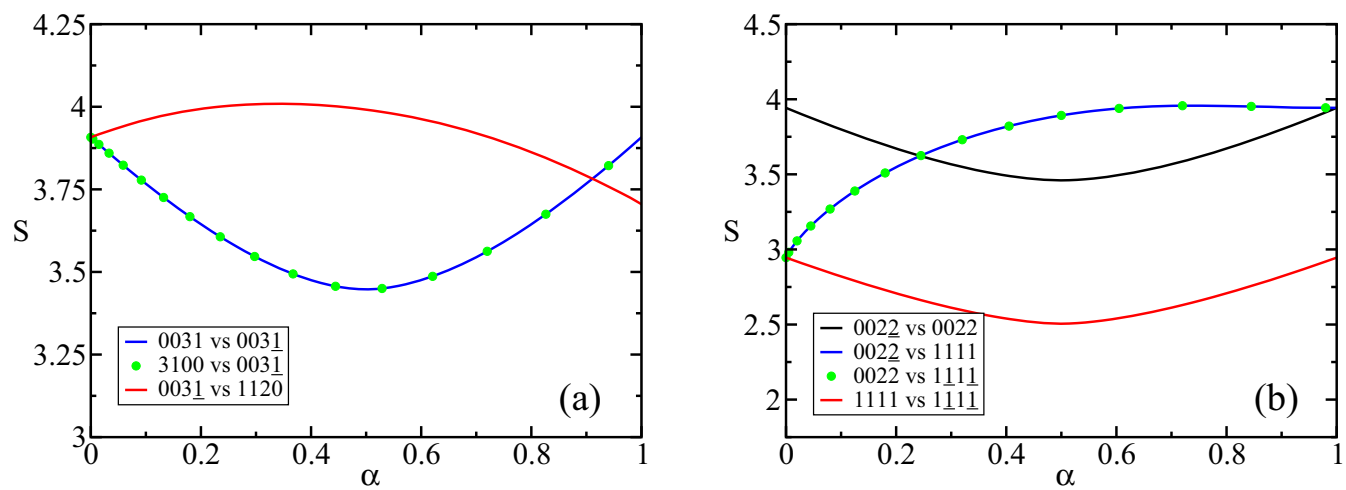

FIG. 1. The von Neumann entropy as a function of the superposition parameter $\alpha$, for states $\left|\psi_{\alpha}\right\rangle=\sqrt{\alpha}|n, m, l, p\rangle+\sqrt{1-\alpha}\left|n^{\prime}, m^{\prime}, l^{\prime}, p^{\prime}\right\rangle$ with $\lambda=0$ (noninteracting oscillators). Note that a negative quantum number, $-n$, is denoted as $\underline{n}$ in the legends. (a) For $|m+p|=1$, the superpositions of states that are shown are $|1,1,2,0\rangle$ with $|0,0,3,-1\rangle$ (red full), $|0,0,3,-1\rangle$ with $|0,0,3,1\rangle$ (blue full), and $|0,0,3,-2\rangle$ with $|3,1,0,0\rangle$ (green dots). (b) For $|m+p|=2$, the superpositions of states that are shown correspond to $|0,0,2,-2\rangle$ with $|0,0,2,2\rangle$ (black full), $|0,0,2,-2\rangle$ with $|1,1,1,1\rangle$ (blue full), $|0,0,2,2\rangle$ with $|1,-1,1,-1\rangle$ (green dots), and $|1,1,1,1\rangle$ with $|1,-1,1,-1\rangle$ (full red). See the corresponding values of $Q_{c}$ in Table I.

representation of a given wave function (see Appendix B) becomes quite taxing. Because of this, it is simpler, and computationally faster, to obtain very accurate approximate variational wave functions using basis set functions that are products of one-particle functions. Once these variational eigenfunctions are calculated they can be used to evaluate matrix representations and approximate eigenvalues of the reduced density matrix corresponding to a given variational eigenfunction. The procedure to calculate each one of these quantities has been described elsewhere (see, for instance, Refs. [22,32] and references therein. The accuracy of the whole procedure can be assessed in different ways, mainly comparing the variational eigenvalues with the corresponding exact values computed using Eq. (24). For all the cases that are discussed in this section the variational eigenvalues corresponding to the variational eigenfunctions used to construct superpositions differ from the exact ones by less than $1 \times 10^{-4}$.

One of the drawbacks of the variational method comes from the fact that assigning quantum numbers to the variational eigenfunctions is usually complicated. In the present case, this assignment is simplified by choosing a particular

TABLE I. Entropies, convexity, and the $Q_{c}$ value for the two cases of noninteracting oscillators, $\lambda=0$, shown in Fig. $1(\mid m+$ $p \mid=1$ and 2). Note that in the first two columns a negative quantum number, $-n$, is denoted as $\underline{n}$.

\begin{tabular}{|c|c|c|c|c|c|c|}
\hline$n m l p$ & $n^{\prime} m^{\prime} l^{\prime} p^{\prime}$ & Convexity & $Q_{c}$ & $S_{v n}$ & $S_{\mathrm{NS}}$ & $S_{R}$ \\
\hline \multicolumn{7}{|c|}{$|m+p|=1$} \\
\hline 0031 & 0031 & Convex & + & 3.907 & 0 & 3.907 \\
\hline $003 \overline{1}$ & 3100 & Convex & + & 3.907 & 0 & 3.907 \\
\hline $112 \overline{0}$ & $003 \underline{1}$ & Concave & - & 3.704 & 1.959 & 1.745 \\
\hline \multicolumn{7}{|c|}{$|m+p|=2$} \\
\hline 0022 & 0022 & Convex & + & 3.94 & 0 & 3.94 \\
\hline $002 \overline{2}$ & 1111 & Concave & - & 3.94 & 2.85 & 1.09 \\
\hline $002 \overline{2}$ & 1111 & Concave & - & 3.94 & 2.85 & 1.09 \\
\hline 1111 & $1 \overline{1} 1 \overline{1}$ & Convex & + & 2.94 & 0 & 2.94 \\
\hline
\end{tabular}

value of the interaction parameter, $\lambda=0.7$, that separates adequately the frequencies $\omega_{R}=1$ and $\omega_{r} \simeq 1.949$, and using basis sets with well-defined values of $L_{z}$. All in all, for the lowest eigenvalues it is possible to unequivocally label the variational eigenfunction using the set of quantum numbers in Eq. (24). The results for the interacting system can then be presented using the same conventions as those used for the noninteracting one. The von Neumann entropy as a function of the superposition parameter is shown in Fig. 2 for several superpositions, and the corresponding values for the remaining entropy, the not-shared one, and the criterion are collected in Table II.

The current example shows all the features that are characteristic of what can be observed in superpositions of degenerate states. The changes in the convexity are correctly predicted by the criterion presented in Sec. II. The example is valuable because it is exactly soluble and its numerical (variational)

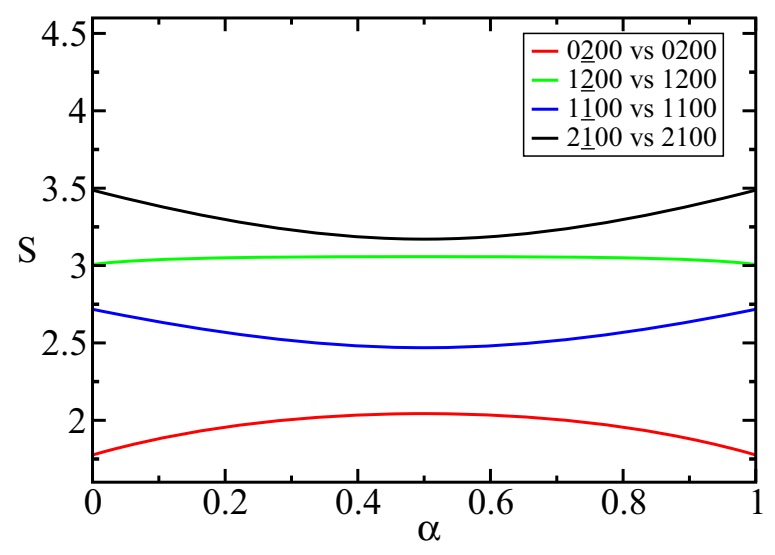

FIG. 2. The von Neumann entropy as a function of the combination parameter $\alpha$ for states $\left|\psi_{\alpha}\right\rangle=\sqrt{\alpha}|n, m, l, p\rangle+$ $\sqrt{1-\alpha}\left|n^{\prime}, m^{\prime}, l^{\prime}, p^{\prime}\right\rangle$ of two interacting oscillators with $\lambda=0.7$ and $|m+p|=1$ and 2 . The combinations of states that are shown are $|0,-2,0,0\rangle$ with $|0,2,0,0\rangle$ (red), $|1,-2,0,0\rangle$ with $|1,2,0,0\rangle$ (green), $|1,-1,0,0\rangle$ with $|1,1,0,0\rangle$ (blue), and $|2,-1,0,0\rangle$ with $|2,1,0,0\rangle$ (black). 
TABLE II. Entropies, convexity, and the $Q_{c}$ value for the two cases of interacting oscillators, $\lambda=0.7$, shown in Fig. $2(|m+p|=$ 1 and 2).

\begin{tabular}{ccccccc}
\hline \hline$n m l p$ & $n^{\prime} m^{\prime} l^{\prime} p^{\prime}$ & Convexity & $Q_{c}$ & $S_{v n}$ & $S_{\mathrm{NS}}$ & $S_{R}$ \\
\hline \multicolumn{7}{c}{$|m+p|=1$} \\
\hline 1100 & 1100 & Convex & + & 2.717 & 1.034 & 1.683 \\
$2 \underline{1} 00$ & 2100 & Convex + & 3.487 & 1.121 & 2.366 \\
\multicolumn{7}{c}{$|m+p|=2$} \\
\hline$\underline{0} 200$ & 0200 & Concave & - & 1.776 & 1.123 & 0.653 \\
$1 \underline{2} 00$ & 1200 & Concave & - & 3.006 & 1.740 & 1.266 \\
\hline \hline
\end{tabular}

implementation is simple. But, to some extent, the peculiar behavior of systems of harmonic oscillators, that can be cast as interacting or noninteracting, limits the scope and validity of the example. So, in Secs. IV and V we present further examples that can be analyzed analytically and present, in the case of the spherium model, strong correlations between the particles.

\section{THE SPHERIUM MODEL}

The number of exactly solvable two interacting particle models in different spatial dimensions is remarkably low, which explains why so many studies of entanglement entropies are about systems of harmonic oscillators or variants of the Calogero model. As has been said in the Introduction, the study of the Calogero model was what triggered the formulation of the criterion that is the object of the present paper. Fortunately, there is a growing number of quasiexactly solvable models [33] that can be used to study properties of strongly interacting two-particle models [34].

The spherium model [35], i.e., two electrons interacting via the Coulomb potential and confined to the surface of a $D$-dimensional sphere, was proposed to study the properties of electronic correlations in a confining geometry. It has been studied using different approaches and as a benchmark to test numerical approximations. As was shown in Ref. [24], this model is quasiexactly solvable, with analytical solutions for particular values of the radius $R$ of the sphere and the dimension $D$. These solutions can be found writing the twoelectron wave function as a bipolar expansion [36], which allows us to calculate eigenfunctions with small total orbital angular momentum numbers, $L=0,1$, or 2 , but it is quite cumbersome to implement for larger values of $L$.

To test the criterion we will construct two-electron wave functions with $L=0,1$, and 2, following the work of Pestka [37], which can be applied in a systematic way. In this section we present the main details of the derivation of the wave functions, the reduced matrix elements, and its eigenvalues.

\section{A. Description of the general solution for $D=3$}

Consider a two-particle Hamiltonian of the form

$$
H=-\frac{1}{2} \nabla_{1}^{2}-\frac{1}{2} \nabla_{2}^{2}+V\left(r_{1}, r_{2}, r_{12}\right)
$$

Note that the potential depends on the radial coordinates of both particles, $r_{1}$ and $r_{2}$, and the distance between them $r_{12}$.
The bipolar decomposition assumes that the solutions that are simultaneous eigenfunctions of the Hamiltonian, the total orbital angular momentum, and the $z$ component of the orbital angular momentum, with eigenvalues $E, L(L+1)$ and $M$, respectively, can be written as

$$
\psi\left(\mathbf{r}_{1}, \mathbf{r}_{2}\right)=\sum_{l_{1}=d_{0}}^{L} \Phi_{l_{1} l_{2}}\left(r_{1}, r_{2}, r_{12}\right) \Omega_{l_{1}, l_{2}}^{L, M}\left(\hat{r}_{1}, \hat{r}_{2}\right),
$$

with the constraint $l_{2}=d-l_{1}$. The inferior limit of the sum must be determined using the parity of the solution $\pi=$ $(-1)^{l_{1}+l_{2}}$, according to

$$
d_{0}=L+1-d \quad \text { and } \quad d= \begin{cases}L+1 & \text { if } \pi=(-1)^{L} \\ L & \text { if } \pi=(-1)^{L+1}\end{cases}
$$

The functions $\Omega_{l_{1}, l_{2}}^{L, M}\left(\hat{r}_{1}, \hat{r}_{2}\right)$ are the eigenfunctions of $\mathbf{L}^{2}$ and $L_{z}$ :

$$
\mathbf{L}^{2} \Omega_{l_{1}, l_{2}}^{L, M}\left(\hat{r}_{1}, \hat{r}_{2}\right)=L(L+1) \Omega_{l_{1}, l_{2}}^{L, M}\left(\hat{r}_{1}, \hat{r}_{2}\right)
$$

and

$$
L_{z} \Omega_{l_{1}, l_{2}}^{L, M}\left(\hat{r}_{1}, \hat{r}_{2}\right)=M \Omega_{l_{1}, l_{2}}^{L, M}\left(\hat{r}_{1}, \hat{r}_{2}\right) .
$$

Note that the wave function in Eq. (28) has $L+1$ or $L$ radial functions (depending on the parity) $\Phi_{l_{1} l_{2}}\left(r_{1}, r_{2}, r_{12}\right)$. Some algebra can be simplified noting that

$$
\begin{array}{rl}
\nabla_{i}^{2} & f\left(r_{1}, r_{2}, r_{12}\right) \Omega_{l_{1}, l_{2}}^{L, M}\left(\hat{r}_{1}, \hat{r}_{2}\right) \\
= & \sum_{\tilde{l}_{1}=d_{0}}^{L}\left\{\hat{X}_{i}^{\tilde{l}_{i}, l_{i}} f\left(r_{1}, r_{2}, r_{12}\right)\right\} \Omega_{\tilde{l}_{1}, \tilde{l}_{2}}^{L, M}\left(\hat{r}_{1}, \hat{r}_{2}\right),
\end{array}
$$

where $\nabla_{i}^{2}$ is any of the Laplacian operators that enter into Eq. (27), and $f\left(r_{1}, r_{2}, r_{12}\right)$ is any function that depends only on the variables $r_{1}, r_{2}$, and $r_{12}$. The curly brackets indicate that the operators $\hat{X}_{i}^{\tilde{l}_{i}, l_{i}}$ are applied only over the radial terms.

The operators $\hat{X}_{i}^{\tilde{l}_{i}, l_{i}}$ have the property that

$$
\hat{X}_{2}^{\tilde{l}, l}\left(r_{1}, r_{2}, r_{12}\right)=\hat{X}_{1}^{\tilde{l}, l}\left(r_{2}, r_{1}, r_{12}\right),
$$

and can be written as

$$
\begin{aligned}
\hat{X}_{i}^{\tilde{l}_{i}, l_{i}}= & \frac{\partial^{2}}{\partial r_{i}^{2}}+\frac{2}{r_{i}} \frac{\partial}{\partial r_{i}}+\frac{\partial^{2}}{\partial r_{12}^{2}} \\
& +\frac{\left(2+l_{i}\right) r_{i}^{2}+l_{i}\left(r_{j}^{2}-r_{12}^{2}\right)}{r_{i}^{2} r_{12}} \frac{\partial}{\partial r_{12}} \\
& +\frac{r_{i}^{2}-r_{j}^{2}+r_{12}^{2}}{r_{i} r_{12}} \frac{\partial^{2}}{\partial r_{i} \partial r_{12}}-\frac{l_{i}\left(l_{i}+1\right)}{r_{i}^{2}},
\end{aligned}
$$

when $0 \leqslant l_{i} \leqslant L$ and

$$
\hat{X}_{i}^{\tilde{l}_{i}-1, l_{i}}=\frac{-2 r_{j}}{r_{i} r_{12}} \sqrt{\frac{\left(L-l_{j}\right)\left(2 l_{i}+1\right)\left(L-l_{i}+1\right)}{2 l_{j}+3}} \frac{\partial}{\partial r_{12}},
$$

for $1 \leqslant l_{i} \leqslant L$. In both cases the value of $j$ is such that $j \neq i$.

When the particles are confined to the surface of a sphere, $r_{1}=r_{2}=R$, the last two expressions can be further simplified, for $0 \leqslant l_{i} \leqslant L$,

$$
\hat{X}_{i}^{\tilde{l}_{i}, l_{i}}=\frac{\partial^{2}}{\partial r_{12}^{2}}+\frac{\left(2+l_{i}\right) R^{2}+l_{i}\left(R^{2}-r_{12}^{2}\right)}{R^{2} r_{12}} \frac{\partial}{\partial r_{12}},
$$


where a constant term is omitted, and for $1 \leqslant l_{i} \leqslant L$,

$$
\hat{X}_{i}^{\tilde{l}_{i}-1, l_{i}}=\frac{-2}{r_{12}} \sqrt{\frac{\left(L-l_{j}\right)\left(2 l_{i}+1\right)\left(L-l_{i}+1\right)}{2 l_{j}+3}} \frac{\partial}{\partial r_{12}} .
$$

All in all, the method proposed by Pestka reduces the calculation of the two-electron wave function to another problem which consists of a set of $L$ (or $L+1$ ) coupled equations for the quantities $\Phi_{l_{1} l_{2}}$.

\section{B. Wave functions with $L=2$}

The wave function with angular momentum number $L=2$ and parity $\pi=(-1)^{L+1}$ depends on just two radial functions, since there are two combinations of possible values: $l_{1}=$ $1, l_{2}=2$ and $l_{1}=2, l_{2}=1$. Using this, we get

$$
\begin{aligned}
& {\left[-\frac{1}{2}\left(\frac{\partial^{2}}{\partial r_{12}^{2}}+\frac{4 R^{2}-r_{12}^{2}}{R^{2} r_{12}} \frac{\partial}{\partial r_{12}}\right)\right.} \\
& \left.\quad-\frac{1}{2}\left(\frac{\partial^{2}}{\partial r_{12}^{2}}+\frac{6 R^{2}-2 r_{12}^{2}}{R^{2} r_{12}} \frac{\partial}{\partial r_{12}}\right)+\frac{1}{r_{12}}-E\right] \Phi_{12} \\
& \quad+\frac{1}{r_{12}} \frac{\partial}{\partial r_{12}} \Phi_{21}=0,
\end{aligned}
$$

and

$$
\begin{aligned}
& {\left[-\frac{1}{2}\left(\frac{\partial^{2}}{\partial r_{12}^{2}}+\frac{4 R^{2}-r_{12}^{2}}{R^{2} r_{12}} \frac{\partial}{\partial r_{12}}\right)\right.} \\
& \left.\quad-\frac{1}{2}\left(\frac{\partial^{2}}{\partial r_{12}^{2}}+\frac{6 R^{2}-2 r_{12}^{2}}{R^{2} r_{12}} \frac{\partial}{\partial r_{12}}\right)+\frac{1}{r_{12}}-E\right] \Phi_{21} \\
& \quad+\frac{1}{r_{12}} \frac{\partial}{\partial r_{12}} \Phi_{12}=0
\end{aligned}
$$

These pairs of coupled equations have a solution of the form $\Phi_{12}=\Phi_{21}=\Phi$. In this case Eqs. (38) and (39) reduce to

$$
\left[\frac{\partial^{2}}{\partial r_{12}^{2}}+\left(\frac{4}{r_{12}}-\frac{3}{2 R^{2}} r_{12}\right) \frac{\partial}{\partial r_{12}}-\frac{1}{r_{12}}+E\right] \Phi=0
$$

Using the ansatz

$$
\Phi=1+r_{12} / \alpha,
$$

we found that this function is a solution with $\alpha=4$ and $R^{2}=$ 6.

Collecting again the partial results, we get the whole set of wave functions with $L=2$ and $M=0, \pm 1, \pm 2$ :

$$
\begin{aligned}
& \Psi_{2, M}\left(\Omega_{1}, \Omega_{2}, r_{12}\right) \\
& \quad=\left[\mathcal{Y}_{1,2}^{2, M}\left(\Omega_{1}, \Omega_{2}\right)-\mathcal{Y}_{1,2}^{2, M}\left(\Omega_{2}, \Omega_{1}\right)\right]\left(1+r_{12} / 4\right),
\end{aligned}
$$

where $\mathcal{Y}_{1,2}^{2, M}\left(\Omega_{1}, \Omega_{2}\right)$ are the usual total angular momentum eigenfunctions defined in terms of the spherical harmonics and the Clebsch-Gordan coefficients.

The one-electron reduced density matrix can be straightforwardly obtained using Perkins's formula [38]:

$$
\begin{aligned}
r_{12}^{k}= & 4 \pi \sum_{l=0}^{L_{1}^{k}}\left(\sum_{m=-l}^{l} Y_{l m}^{*}\left(\Omega_{1}\right) Y_{l m}\left(\Omega_{2}\right)\right) \\
& \times\left(\sum_{t=0}^{L_{2}^{k, l}} C_{k l t} r_{<}^{l+2 t} r_{>}^{k-(l+2 t)}\right) .
\end{aligned}
$$

This expression is valid for $k=-1,0,1, \ldots$, and the coefficients $L_{1}^{k}$ and $L_{2}^{k, l}$ are given by

$$
L_{1}^{k}=\left\{\begin{array}{lll}
\frac{k}{2} & \text { if } & k \text { is even } \\
\infty & \text { if } & k \text { is odd }
\end{array},\right.
$$

and

$$
L_{2}^{k, l}=\left\{\begin{array}{ccc}
\frac{k}{2}-l & \text { if } & k \text { is even } \\
\frac{k+1}{2} & \text { if } & k \text { is odd }
\end{array} .\right.
$$

The coefficients $C_{k l t}$ are given by

$$
C_{k l t}=\left\{\begin{array}{lll}
\frac{1}{k+2}\left(\begin{array}{l}
k+2 \\
2 t+1
\end{array}\right) & \text { if } \quad l=0 \\
\frac{1}{k+2}\left(\begin{array}{l}
k+2 \\
2 t+1
\end{array}\right) \prod_{\alpha=0}^{\min [l-1,(k+1) / 2]} \frac{2 t-k+2 \alpha}{2 t+1+2 l-2 \alpha} & \text { if } \quad l>0
\end{array} .\right.
$$

From Eqs. (41) and (43) it is clear that the wave functions can be completely written in terms of spherical harmonics of both solid angles, $Y_{l m}\left(\Omega_{1}\right)$ and $Y_{l m}\left(\Omega_{2}\right)$.

So, if $\Psi(1,2)$ is any given superposition of two-electron wave functions, the corresponding reduced density matrix is

$$
\rho\left(1,1^{\prime}\right)=\int \Psi^{*}(1,2) \Psi\left(1^{\prime}, 2\right) d \Omega_{2} .
$$

To calculate the necessary eigenvalues, the matrix representation of the density operator in Eq. (47) is calculated in a basis. It is natural to use the spherical harmonics

$$
[\rho]_{j, s, j^{\prime}, s^{\prime}}=\int d \Omega_{1} \int d \Omega_{1^{\prime}} Y_{j, s}^{*}(1) \rho\left(1,1^{\prime}\right) Y_{j^{\prime}, s^{\prime}}\left(1^{\prime}\right) .
$$

Fortunately, to evaluate explicitly and exactly both Eqs. (47) and (48) only integrals involving two and three spherical harmonics are required:

$$
\begin{aligned}
\int Y_{l_{3}, m_{3}}^{*}(\theta, \varphi) Y_{l_{2}, m_{2}}(\theta, \varphi) Y_{l_{1}, m_{1}}(\theta, \varphi) d \Omega \\
=\left[\frac{\left(2 l_{1}+1\right)\left(2 l_{2}+1\right)}{4 \pi\left(2 l_{3}+1\right)}\right]^{1 / 2} \\
\quad \times C\left(l_{1}, m_{1} ; l_{2}, m_{2} ; l_{3}, m_{3}\right) C\left(l_{1}, 0 ; l_{2}, 0 ; l_{3}, 0\right),
\end{aligned}
$$




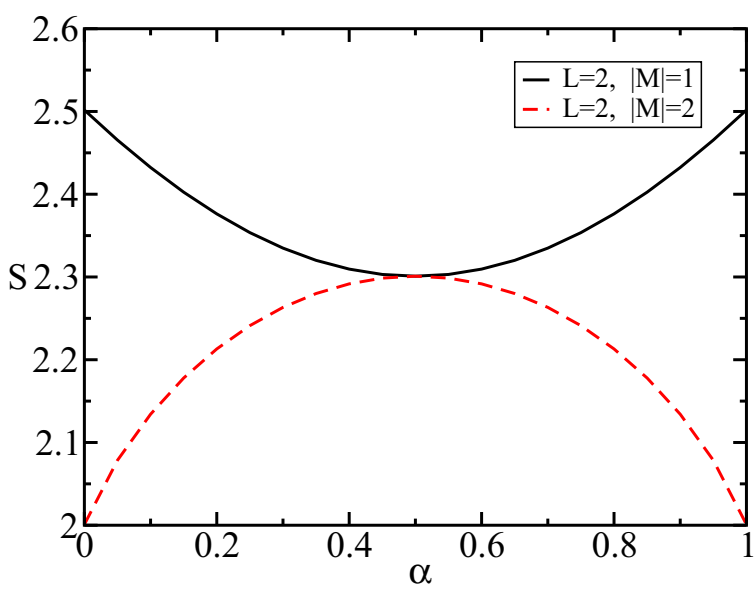

FIG. 3. The von Neumann entropy as a function of $\alpha$ for $L=2$ states of two electrons on a surface of a sphere. The states are a linear combination of the form shown in Eq. (51). The entropy with $|M|=$ 1 is depicted with the black line and that with $|M|=2$ is depicted with a red dashed line. In Table III the values of the entropies, the criterion prediction, and convexities of the curves are shown.

together with the formula

$$
\begin{aligned}
& Y_{l_{1}, m_{1}}(\theta, \varphi) Y_{l_{2}, m_{2}}(\theta, \varphi) \\
& =\sum_{L=0}^{\infty}\left[\frac{\left(2 l_{1}+1\right)\left(2 l_{2}+1\right)}{4 \pi(2 L+1)}\right]^{1 / 2} \\
& \quad \times C\left(l_{1}, m_{1} ; l_{2}, m_{2} ; L, M\right) C\left(l_{1}, 0 ; l_{2}, 0 ; L, 0\right) \\
& \quad \times Y_{L, m_{1}+m_{2}}(\theta, \varphi) .
\end{aligned}
$$

Figure 3 shows the results for the $L=2$ case. The superposition state $\left|\psi_{\alpha}\right\rangle$ of Eq. (8) is in this case

$$
\left|\psi_{\alpha}\right\rangle=\sqrt{\alpha}\left|\Psi_{L, M}\right\rangle+\sqrt{1-\alpha}\left|\Psi_{L,-M}\right\rangle .
$$

As can be seen in Fig. 3, the two possible elections for $|M|=$ 1,2 render a convex and a concave entropy curve for the superposition state $\left|\psi_{\alpha}\right\rangle$, respectively. The criterion, shown in Table III, correctly predicts the convexity in both cases.

\section{LAGUERRE-GAUSSIAN ONE-PHOTON STATES}

Two-photon states can be constructed, from a theoretical point of view, just applying two creation operators to the vacuum state. From an experimental point of view, the most used method is the spontaneous parametric down conversion (SPDC) [26]. It is well known that the SPDC mechanism provides a couple of photons in an entangled state that can be used to perform different quantum information tasks. Nevertheless, the two-photon state depends on the mode function of the pump and the phase-matching conditions. So, to analyze

TABLE III. The values of the convexity, the criterion prediction, and entropies of the curves shown in Fig. 3.

\begin{tabular}{lccccc}
\hline \hline$L M$ & $L M^{\prime}$ & Convexity & $Q_{c}$ & $S_{\mathrm{NS}}$ & $S_{R}$ \\
\hline 21 & $2 \underline{1}$ & Convex & + & 0.917 & 1.584 \\
22 & $2 \underline{2}$ & Concave & - & 1.422 & 0.578 \\
\hline \hline
\end{tabular}

a simpler case we focus on one-photon states and use the concept of single-particle entanglement [28] where one spatial degree of freedom of the photon wave function is traced out. Since we are interested in states that are eigenstates of $L_{z}$ the Laguerre-Gaussian states are an obvious choice to test the criterion. They are given by [26,28]

$$
\begin{aligned}
& u_{l m}^{\mathrm{LG}}(k ; \vec{r}, t) \\
& \quad=\frac{4 \pi(-1)^{l+|m|} l !}{s^{2(l+|m|+1)}(z)} r^{|m|} e^{i m \phi} L_{l}^{|m|}\left(\frac{r^{2}}{s^{2}(z)}\right) e^{i k z-i \omega_{k} t-r^{2} / s^{2}(z)},
\end{aligned}
$$

where $r, \phi, z$ are the usual cylindrical coordinates, $m$ is the quantum number of the $z$ component of the orbital angular momentum, $L_{l}^{|m|}$ are the modified Laguerre polynomials, and the waist function $s(z)$ is a classical quantity that quantifies the width of the beam along the $z$ direction:

$$
s^{2}(z)=s_{0}^{2}+i \frac{2 z}{k} .
$$

In the following we drop the superscript in $u_{l m}^{\mathrm{LG}}$ to simplify the notation and make the change of variable $x=r / s(z)$. Let us recall that the Laguerre-Gaussian states are transverse modes that describe the free propagation of a photon with energy $\hbar \omega_{k}$, and are solutions of the Helmholtz equation in the paraxial approximation.

At this stage the procedure and the quantities to be calculated are well known so, in this section, we include which states are studied and the formal expression for the reduced density matrix.

For a superposition given by

$$
\left|\psi_{\alpha}\right\rangle=\sqrt{\alpha}\left|u_{l m}\right\rangle+\sqrt{1-\alpha}\left|u_{l^{\prime} m^{\prime}}\right\rangle,
$$

where both $l, m$ and $l^{\prime}, m^{\prime}$ are quantum numbers compatible with Eq. (52), we calculate the reduced density matrix:

$$
\begin{aligned}
\rho_{\text {red }}\left(x, x^{\prime}\right)= & \int_{-\infty}^{\infty} d y\left\{\alpha u_{l m}^{*}(x, y, z) u_{l m}\left(x^{\prime}, y, z\right)\right. \\
& +(1-\alpha) u_{l^{\prime} m^{\prime}}^{*}(x, y, z) u_{l^{\prime} m^{\prime}}\left(x^{\prime}, y, z\right) \\
& +\sqrt{\alpha(1-\alpha)}\left[u_{l m}^{*}(x, y, z) u_{l^{\prime} m^{\prime}}\left(x^{\prime}, y, z\right)\right. \\
& \left.\left.+u_{l^{\prime} m^{\prime}}^{*}(x, y, z) u_{l m}\left(x^{\prime}, y, z\right)\right]\right\},
\end{aligned}
$$

where $z$ is considered as a parameter and $x$ and $y$ are the usual Cartesian coordinates perpendicular to $z$. We consider reduced density matrices at constant values of $z$ since the LG states in Eq. (52) are not square-integrable functions. To avoid this kind of assumption it is possible to implement the calculation of reduced density matrices and the corresponding entropies using LG modes in a cavity [39,40], which are square integrable and very similar to those in Eq. (52), so the dependency on the $z$ variable can be traced out completely.

To test the criterion it is necessary to obtain the matrix elements

$$
\left[\rho_{\mathrm{red}}\right]_{a b}=\int_{-\infty}^{\infty} d x \int_{-\infty}^{\infty} d x^{\prime} f_{a}(x) \rho_{\mathrm{red}}\left(x, x^{\prime}\right) f_{b}\left(x^{\prime}\right),
$$




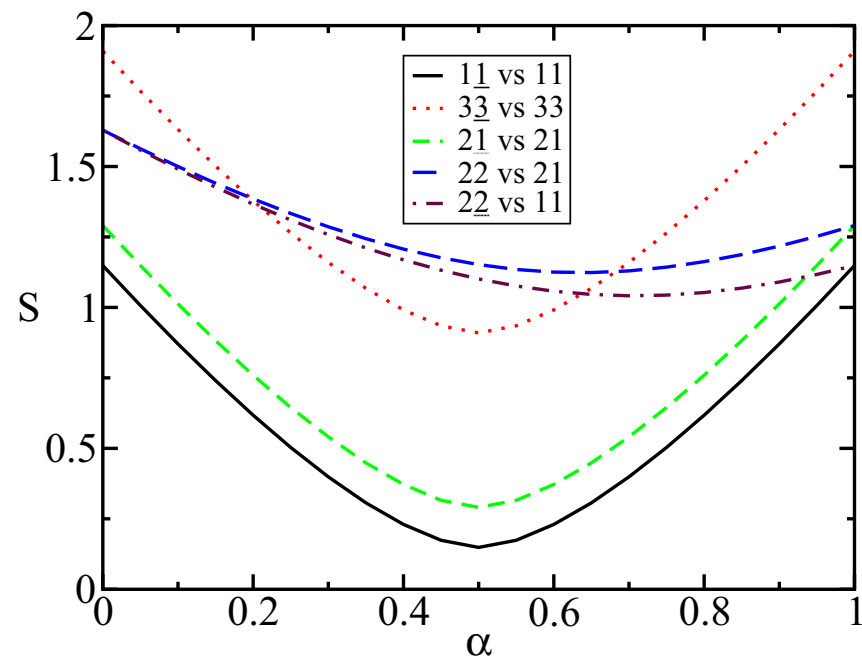

FIG. 4. The von Neumann entropy as a function of the combination parameter $\alpha$, for states $\left|\psi_{a}\right\rangle$ from Eq. (54). The symmetric combinations $\left(l=l^{\prime}\right.$ and $\left.m=-m^{\prime}\right)\left(l_{\alpha=0}, m_{\alpha=0}, l_{\alpha=1}, m_{\alpha=1}\right)=$ $(1,-1,1,1),(3,-3,3,3)$, and $(2,-1,2,1)$ are shown with black, red pointed, and green dashed curves, respectively. The asymmetric combinations $\left(l_{0}, m_{0}, l_{1}, m_{1}\right)=(2,2,2,1),(2,-2,1,1)$ are shown with blue dashed and brown dash-dotted curves, respectively.

where the one-coordinate basis functions used are the Hermite functions

$$
f_{a}(x)=\frac{1}{\sqrt{\sqrt{\pi} 2^{a} a !}} H_{a}(x) e^{-x^{2} / 2}, \quad a=0,1,2 \ldots
$$

Figure 4 shows the results obtained for these states. As the results included in Table IV show, the criterion correctly predicts the convexity of the superpositions considered.

The one-photon eigenstates cannot provide a superposition with a concave von Neumann entropy because the one-photon wave function depends on the two transversal coordinates, $x$ and $y$, in exactly the same way. It is clear from Eq. (52) that the state with quantum number $m$ depends on the same set of Laguerre polynomials as the state with quantum number $-m$. So, when tracing one or the other coordinates the corresponding reduced density matrices that enter into the calculation of the criterion both "occupy" the same portion of the one-coordinate Hilbert space. As a consequence their remaining entropy always overcomes their not-shared entropy.

TABLE IV. The values of the convexity, the criterion prediction, and the entropies of the curves shown in Fig. 4.

\begin{tabular}{cccccc}
\hline \hline$l m$ & $l^{\prime} m^{\prime}$ & Convexity & $Q_{c}$ & $S_{\mathrm{NS}}$ & $S_{R}$ \\
\hline 11 & $1 \underline{1}$ & Convex & + & 0 & 0.796 \\
21 & $2 \underline{1}$ & Convex & + & 0 & 0.894 \\
21 & $2 \frac{2}{2}$ & Convex & + & 0.194 & 0.700 \\
11 & $2 \underline{2}$ & Convex & + & 0.187 & 0.608 \\
33 & $3 \underline{3}$ & Convex & + & 0 & 1.323 \\
\hline \hline
\end{tabular}

\section{TWO-PARTICLE TOTAL ANGULAR MOMENTUM EIGENSTATES}

So far, the examples analyzed in the previous sections provide a strong evidence of the validity of the criterion stated in this paper about the convexity of superpositions of degenerate states. Regrettably, all of them have reduced density matrices $\rho_{A, 0}$ with nondegenerate eigenvalues.

An exact example showing reduced density matrices with degenerate eigenvalues can be constructed from the addition of two angular momentum operators. As usual we consider

$$
\begin{aligned}
& \mathbf{L}^{2}\left|L M ; l_{1}, l_{2}\right\rangle=L(L+1)\left|L M ; l_{1}, l_{2}\right\rangle, \\
& L_{z}\left|L M ; l_{1}, l_{2}\right\rangle=M\left|L M ; l_{1}, l_{2}\right\rangle,
\end{aligned}
$$

where $\mathbf{L}^{2}=\left(\mathbf{L}_{1}+\mathbf{L}_{2}\right)^{2}, L_{z}=L_{z}^{(1)}+L_{z}^{(2)}$. For each particle the square of the angular momentum operator, $\mathbf{L}_{i}^{2}$, and its $z$ component, $L_{z}^{(i)}$, have common eigenfunctions which satisfy

$$
\mathbf{L}_{i}^{2}\left|l_{i}, m_{i}\right\rangle=l_{i}\left(l_{i}+1\right)\left|l_{i}, m_{i}\right\rangle, \quad L_{z}^{i}\left|l_{i}, m_{i}\right\rangle=m_{i}\left|l_{i}, m_{i}\right\rangle,
$$

for $i=1,2$.

To refine our concepts, let us consider the Hamiltonian for two interacting spins given by

$$
H=\mathbf{L}^{2}-L_{z}^{2}=\mathbf{L}_{1}^{2}+\mathbf{L}_{2}^{2}+2 \mathbf{L}_{1} \cdot \mathbf{L}_{2}-L_{z}^{2}
$$

and choose two spins with the same angular quantum number, $l_{1}=l_{2}=\ell$. Consistently with the superpositions analyzed in previous sections, we consider states given by

$$
\Phi=\sqrt{\alpha} \mathcal{Y}_{\ell, \ell}^{L, L}+\sqrt{1-\alpha} \mathcal{Y}_{\ell, \ell}^{L,-L}
$$

where

$$
\mathcal{Y}_{\ell, \ell}^{L, \pm L}=\sum_{m_{1}, m_{2}} C\left(\ell, m_{1} ; \ell, m_{2} ; L, \pm L\right) Y_{\ell, m_{1}}\left(\Omega_{1}\right) Y_{\ell, m_{2}}\left(\Omega_{2}\right)
$$

and $C\left(\ell, m_{1} ; \ell, m_{2} ; L, M\right)$ are the Clebsch-Gordan coefficients.

The states $\mathcal{Y}_{\ell, \ell}^{L, L}$ and $\mathcal{Y}_{\ell, \ell}^{L,-L}$ are degenerate since

$$
H \mathcal{Y}_{\ell, \ell}^{L, \pm L}=L \mathcal{Y}_{\ell, \ell}^{L, \pm L}
$$

and for fixed $L$ they are the states with minimum energy.

We include in Appendix A the necessary algebraic details to evaluate explicitly and exactly the entries of the different reduced density matrices and their eigenvalues. Figure 5(a) shows the explicit evaluation of $S\left(\rho_{A, \alpha}\right)$ for the case $\ell=$ 3, $|M|=L$, and $L=1,2,3,4,5$ and 6. It can be appreciated that the cases $L=3,4,5$, and 6 have a different convexity than the $L=1$ and 2 cases. On the other hand, the solid circular dots in Fig. 5(b) correspond to the values of the not-shared and remaining entropies calculated using Eqs. (12) and (15). The lines are included as a guide to the eye. It is clear that using these entropies the criterion detects the correct convexity of all the cases, as it is shown in Table V.

It is interesting to analyze in some detail the case $\ell=3$ and $L=2$. The reduced density matrices can be calculated explicitly and, in the standard one-particle angular momentum basis $\{|m=3\rangle,|m=2\rangle, \ldots,|m=-3\rangle\}$, they are diagonal matrices:

$$
\rho_{A, 0}=\operatorname{diag}\left\{\frac{5}{42}, \frac{5}{21}, \frac{2}{7}, \frac{5}{21}, \frac{5}{42}, 0,0\right\},
$$



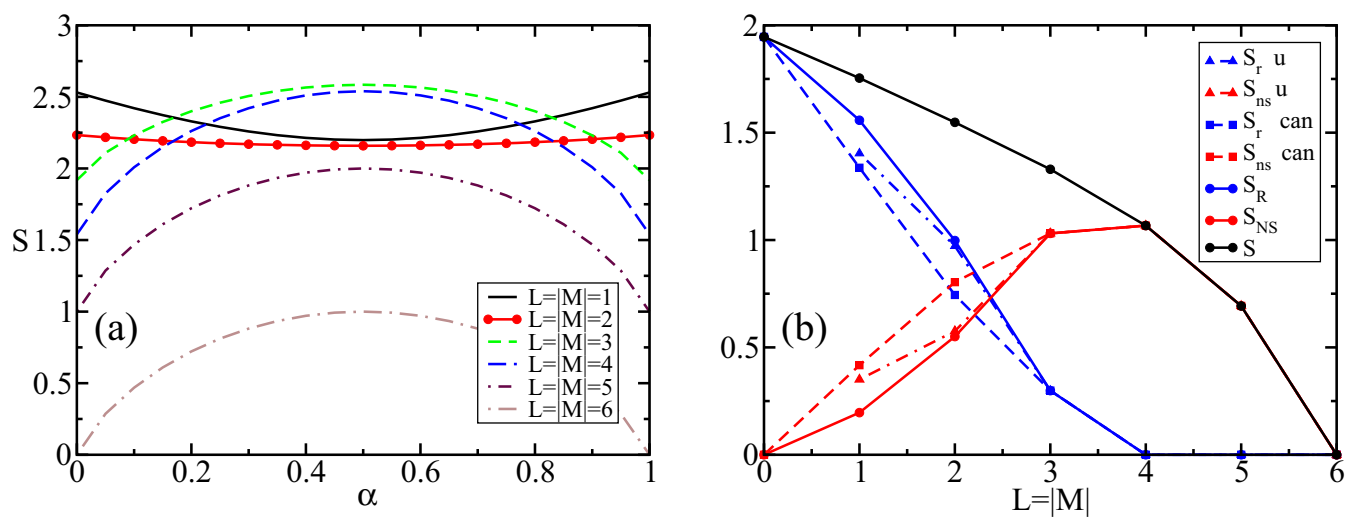

FIG. 5. (a) The von Neumann entropy as a function of the combination parameter $\alpha$, for the states of Eq. (61) with $\ell=3$ and $L=|M|$. $|M|$ and $L$ values for each curve are given in the legend. (b) The von Neumann, remaining, and not-shared entropies calculated for the cases shown in (a) are shown using black, red, and blue solid circular dots, respectively. The square dots correspond to the not-shareable entropy calculated using the canonical angular momentum basis and the triangular ones correspond to the entropy calculated using another basis (see the text for details).

and

$$
\rho_{A, 1}=\operatorname{diag}\left\{0,0, \frac{5}{42}, \frac{5}{21}, \frac{2}{7}, \frac{5}{21}, \frac{5}{42}\right\} \text {. }
$$

It is clear that the matrix $\rho_{A, 0}$ has two pairs of degenerate eigenvalues, the first and fifth one are the first pair $(\lambda=5 / 42)$, and the second and fourth are the second one $(\lambda=5 / 21)$. Accordingly with Eq. (14), to contribute to the not-shared entropy we must compare two times the eigenvalue of $\rho_{A, 0}$ with the sum of eigenvalues of $\rho_{A, 1}$ that lie in the same subspace. But, since $2 \times \frac{5}{42}=\frac{5}{6} \times \frac{2}{7}<\frac{2}{7}$,

$$
\min \left\{\sum_{i=1}^{2} \Theta\left[\lambda_{v}^{A, 0}-\left\langle\rho_{A, 1}\right\rangle_{i, v}\right] \log _{2}\left(\frac{1}{\lambda_{v}^{A, 0}}\right)\right\}=0 .
$$

On the other hand, the term associated to the eigenvalue $5 / 21$ contributes with

$$
\tilde{S}=\left(2 \times \frac{5}{21}-\frac{5}{21}\right) \log _{2} \frac{21}{5} .
$$

Collecting these results, the not-shared entropy is equal to

$$
S_{\mathrm{NS}}=\left(\frac{2}{7}-\frac{5}{42}\right) \log _{2} \frac{7}{2}+\left(\frac{5}{21}\right) \log _{2} \frac{21}{5} .
$$

It is instructive, and simple to do, to check what happens if the minimization implied in the definition of the not-shared entropy is not performed, i.e., what values are obtained for

TABLE V. The values of the convexity, the criterion prediction, and the entropies of the curves shown in Fig. 5.

\begin{tabular}{cccccc}
\hline \hline$L$ & $|M|$ & Convexity & $Q_{c}$ & $S_{\mathrm{NS}}$ & $S_{R}$ \\
\hline 1 & 1 & Convex & + & 0.196 & 1.558 \\
2 & 2 & Convex & + & 0.550 & 0.997 \\
3 & 3 & Concave & - & 1.031 & 0.299 \\
4 & 4 & Concave & - & 1.067 & 0 \\
5 & 5 & Concave & - & 0.693 & 0 \\
6 & 6 & Concave & 0 & 0 & 0 \\
\hline \hline
\end{tabular}

different choices of the one-dimensional projectors associated to the degenerate eigenvalues. Choosing the canonical one-particle angular momentum basis to generate the onedimensional projectors gives the not-shareable entropy:

$$
S_{\mathrm{ns}}=-\sum_{\lambda_{i}^{A, 0} \neq 0}^{7} \Theta\left[\lambda_{i}^{A, 0}-\lambda_{i}^{A, 1}\right] \log _{2}\left(\lambda_{i}^{A, 0}\right),
$$

where the eigenvalues, for the case $L=2$, are those in Eqs. (64) and (65). The values obtained using Eq. (69) are shown in Fig. 5(b) as square solid blue dots. Not surprisingly, the values are larger than those of $S_{\mathrm{NS}}$, but, more interestingly, it is clear that for $L=2$ the not-shareable entropy is larger than the remaining one, which could lead to an incorrect assessment of the convexity. Sometimes, choosing a particular basis to obtain the projectors $P_{i}^{A, 0}$ could give very good results for particular values of $\ell$ and $|M|=L$; for instance, in Fig. 5 the values obtained using a particular basis are shown using triangular dots. This election provides values of the not-shareable entropy that are larger than those of $S_{\mathrm{NS}}$ and predicts correctly the curvature.

We have tested a very large number of cases, up to $\ell=12$, which can be done quite quickly and efficiently given the simplicity of the bipartite states $\mathcal{Y}_{\ell, \ell}^{L, M}$, and for all these cases the criterion predicts correctly the curvature of the superposition of states.

\section{DISCUSSION AND CONCLUSIONS}

From a theoretical point of view, the amount of analytical work involved in the examples presented, the two twodimensional harmonic oscillators and the spherium, indicates how difficult it is to construct exact cases to test the convexity criterion. All the quantities involved, in particular the matrix elements of the reduced density matrices, involve a large number of nested sums, so its evaluation time grows as $M^{\text {nes+1 }}$, where nes is the number of nested sums and $M$ is the largest one-particle basis set size that it is necessary to use in order to guarantee the normalization of the reduced density matrix. 
The criterion could be tested using pure states of manybody models (spin chains) which, in some cases, have exact solutions. The reduced matrices can be obtained using the adequate spin-correlation functions for small subsystems. Since so far we have studied only bipartite systems made of two subsystems the Hilbert spaces of which have the same dimension it is not clear if some amendments are in order for the criterion to work in the spin chain setting. Work along this line is in progress.

Our results imply that, most likely, there should be a theorem about the convexity of the von Neumann entropy of superpositions of pure states but, so far, we have not been able to formulate the precise hypotheses that make it work, i.e., we know that the superpositions of pure degenerate eigenstates satisfy the requirements to have a defined convexity but we do not have an algorithm that allows us to generate a number of eigenvalues or eigenfunctions (or projectors) to construct $\rho_{A, 0}$ and $\rho_{A, 1}$ and $\rho_{A, \alpha}$ and guarantee that $S\left(\rho_{A, \alpha}\right)$ will be convex (or concave). In this sense, the condition that the superposition is made of two degenerate states with quantum number $\pm m$, where $m$ is the quantum number associated to the $z$ component of the total angular momentum, seems to be sufficient for states that are defined over hyperspheres or that have the same asymptotic behavior as the harmonic oscillator eigenfunctions.

In the same sense as in the paragraph above, it is not necessary that $S\left(\rho_{A, 0}\right)=S\left(\rho_{A, 1}\right)$ to ensure that the von Neumann entropy, $S\left(\rho_{A, \alpha}\right)$, has a well-defined convexity (concavity). Nevertheless, it is worth pointing out that if $\rho_{A, 0}=$ $\operatorname{Tr}_{B}\left(\left|\psi_{L}\right\rangle\left\langle\psi_{L}\right|\right)$ or $\rho_{A, 0}=\operatorname{Tr}_{B}\left(\left|\psi_{-L}\right\rangle\left\langle\psi_{-L}\right|\right)$ the criterion predicts exactly the same convexity (concavity). In other words, the criterion holds even when $\rho_{A, 0}$ and $\rho_{A, 1}$ are not isospectral.

In contradistinction to what happens to the entropy of one-photon states, for the eigenstates of the total angular momentum it is possible to find concave and convex functions. For example, for the states $|2 \ell, 2 \ell, \ell, \ell\rangle$ and $|2 \ell,-2 \ell, \ell \ell\rangle$, once a particle is traced out, they result in orthogonal states that do not share entropy and, consequently, the von Neumann entropy of the superposition is concave. Also, for some value $k$ the states $\operatorname{Tr}_{B}(|2 \ell-k, 2 \ell-k, \ell, \ell\rangle\langle 2 \ell-k, 2 \ell-$ $k, \ell, \ell \mid)$ and $\operatorname{Tr}_{B}(|2 \ell-k,-(2 \ell-k), \ell, \ell\rangle\langle 2 \ell-k,-(2 \ell-$ $k), \ell, \ell \mid)$ are no longer orthogonal and share some entropy. At some larger value $k_{c}>k$ the remaining entropy overcomes the not-shared entropy and the von Neumann entropy $S\left(\rho_{A, \alpha}\right)$ becomes convex.

In Ref. [22] it was envisaged that certain superpositions of degenerate bipartite states could have definite convexity (concavity) and that the extremal states would also be eigenstates of other observables of the system [22], and it was required that the system under study had at least two conserved quantities. In this paper we have restricted ourselves to the case where those quantities are the energy and the $z$ component of the total angular momentum, which is very reasonable for systems with a preferred direction. For particle systems it is difficult to construct other conserved quantities beyond the Hamiltonian, the total angular momentum, or some of its components, unless some superintegrable system is considered. There are some examples of two- and three-body superintegrable problems in two and three dimensions, where the conserved quantities are polynomials of the momentum operator Cartesian components. Currently, we are studying the convexity properties of superpositions of degenerate states in this kind of problems.

As a final comment, we want to return to a subject that we raised at the end of Sec. II, where we stated that it could be desirable to formulate the not-shared entropy, Eq. (12), without resorting to the spectral decompositions of the reduced density operators. Here, we discuss some numerical tests that we implemented in the examples considered in Sec. VI, i.e., orbital angular momentum states with quantum number $L$. Consider a set of one-dimensional projectors $\left\{P_{\alpha}\right\}_{\alpha=1}^{2 L+1}$ that are mutually orthogonal such that $\sum_{\alpha} P_{\alpha}=I$. Also, consider the quantity

$$
\bar{S}=-\sum_{\alpha} \max \left[\left\langle\rho_{A, 0}\right\rangle_{\alpha}-\left\langle\rho_{A, 1}\right\rangle_{\alpha}, 0\right] \log _{2}\left(\left\langle\rho_{A, 0}\right\rangle_{\alpha}\right),
$$

where $\left\langle\rho_{A, i}\right\rangle_{\alpha}=\operatorname{Tr}\left(\rho_{A, i} P_{\alpha}\right)$. For very small values of $L$ it is numerically feasible to show that

$$
\min (S-2 \bar{S})=S-2 S_{\mathrm{NS}},
$$

where the minimum was obtained generating randomly families of orthogonal projectors and evaluating $\bar{S}$. For larger values of $L$ the number of random families of projectors necessary to pick up approximately the value of the minimum grows so fast that a more educated sampling becomes mandatory. Choosing random sets of projectors close enough to the eigenprojectors of $\rho_{A, 0}$, Eq. (70), was verified for moderate values of $L$ such as the ones studied in Sec. VI. Note that if for a set of projectors $S-2 \bar{S}<0$ then this is sufficient to affirm that the superposition will be concave. Further work along these lines is under progress.

\section{ACKNOWLEDGMENTS}

We acknowledge CONICET (Grant No. PIP$11220150100327 \mathrm{CO})$ for partial financial support. N.G. and O.O. also acknowledge Secretaria de Ciencia y Tecnología-Universidad Nacional de Córdoba for partial financial support.

\section{APPENDIX A: ANGULAR MOMENTUM REDUCED DENSITY MATRICES EXAMPLE}

The simpler example that allows us to construct bipartite states to test if the von Neumann entropy of a given superposition $S\left(\rho_{A, \alpha}\right)$ is a convex function can be constructed from the states

$$
\mathcal{Y}_{l_{1}, l_{2}}^{L, M}=\sum_{m_{1}, m_{2}} C\left(l_{1}, m_{1} ; l_{2}, m_{2} ; L, M\right) Y_{l_{1}, m_{1}}\left(\Omega_{1}\right) Y_{l_{2}, m_{2}}\left(\Omega_{2}\right),
$$


where $C\left(l_{1}, m_{1} ; l_{2}, m_{2} ; L, M\right)$ are the Clebsch-Gordan coefficients and $Y_{l_{1}, m_{1}}$ are the usual spherical harmonics. For superpositions of the form

$$
\Phi=\sqrt{\alpha} \mathcal{Y}_{l_{1}, l_{2}}^{L, M}+\sqrt{1-\alpha} \mathcal{Y}_{l_{1}, l_{2}}^{L,-M}
$$

the reduced density operator is

$$
\begin{aligned}
\rho_{\text {red }}^{\alpha}\left(\Omega_{1}, \Omega_{1}^{\prime}\right)= & \int \Phi^{*}\left(\Omega_{1}, \Omega_{2}\right) \Phi\left(\Omega_{1}^{\prime}, \Omega_{2}\right) d \Omega_{2}=\int\left\{\alpha \mathcal{Y}_{l_{1}, l_{2}}^{* L, M}\left(\Omega_{1}, \Omega_{2}\right) \mathcal{Y}_{l_{1}, l_{2}}^{L, M}\left(\Omega_{1}^{\prime}, \Omega_{2}\right)\right. \\
& +(1-\alpha) \mathcal{Y}_{l_{1}, l_{2}}^{* L, M}\left(\Omega_{1}, \Omega_{2}\right) \mathcal{Y}_{l_{1}, l_{2}}^{L, M}\left(\Omega_{1}^{\prime}, \Omega_{2}\right)+\sqrt{\alpha(1-\alpha)}\left[\mathcal{Y}_{l_{1}, l_{2}}^{* L, M}\left(\Omega_{1}, \Omega_{2}\right) \mathcal{Y}_{l_{1}, l_{2}}^{L,-M}\left(\Omega_{1}^{\prime}, \Omega_{2}\right)\right. \\
& \left.\left.+\mathcal{Y}_{l_{1}, l_{2}}^{* L,-M}\left(\Omega_{1}, \Omega_{2}\right) \mathcal{Y}_{l_{1}, l_{2}}^{L, M}\left(\Omega_{1}^{\prime}, \Omega_{2}\right)\right]\right\} d \Omega_{2} .
\end{aligned}
$$

The reduced density matrix above is a function of both solid angles $\Omega_{1}$ and $\Omega_{1}^{\prime}$, so it is logical to look for its expression in the spherical harmonics basis, where its elements are given by

$$
\left[\rho_{\text {red }}^{\alpha}\right]_{i, j}=\int Y_{l_{1}, i}\left(\Omega_{1}\right) \rho_{\text {red }}^{\alpha}\left(\Omega_{1}, \Omega_{1}^{\prime}\right) Y_{l_{1}, j}^{*}\left(\Omega_{1}^{\prime}\right) d \Omega_{1} d \Omega_{1}^{\prime}
$$

After some tedious but straightforward algebra, it can be shown that

$$
\begin{aligned}
{\left[\rho_{\text {red }}^{\alpha}\right]_{i, j}=} & \sum_{m_{2}}\left\{\alpha C\left(l_{1}, i ; l_{2}, m_{2} ; L, M\right) C\left(l_{1}, j ; l_{2}, m_{2} ; L, M\right)+(1-\alpha) C\left(l_{1}, i ; l_{2}, m_{2} ; L,-M\right) C\left(l_{1}, j ; l_{2}, m_{2} ; L,-M\right)\right. \\
& \left.+\sqrt{\alpha(1-\alpha)}\left[C\left(l_{1}, i ; l_{2}, m_{2} ; L, M\right) C\left(l_{1}, j ; l_{2}, m_{2} ; L,-M\right)+C\left(l_{1}, i ; l_{2}, m_{2} ; L,-M\right) C\left(l_{1}, j ; l_{2}, m_{2} ; L, M\right)\right]\right\}
\end{aligned}
$$

where $|i|,|j| \leqslant l_{1}$.

\section{APPENDIX B: TWO HARMONIC OSCILLATORS RELATED EXPRESSIONS}

In this Appendix we present explicit expressions of the two harmonic oscillator states that allow the computation of the density matrix eigenvalues. The angular momentum two-particle eigenstates in Eqs. (24) and (25) can be written as

$$
|n, m, l, p\rangle=|n, m\rangle_{R, \phi_{R}}|l, p\rangle_{r, \phi_{r}}
$$

where the subindices indicate for which oscillator the vector sate is an eigenstate, following the convention that $R$ designates the centered coordinates oscillator and $r$ designates the relative coordinates one.

Each one of the one-particle angular momentum eigenvectors in Eq. (B1) can be written as a linear combination of Cartesian oscillator states, $\left|n_{x}, n_{y}\right\rangle^{c}=\left|n_{x}\right\rangle^{c}\left|n_{y}\right\rangle^{c}[31]$, as follows:

$$
\begin{aligned}
& |n, m\rangle_{\tilde{r}, \tilde{\phi}}=\frac{1}{\sqrt{n !(n+|m|) ! 2^{(2 n+|m|) / 2}}} \sum_{j=0}^{n} \sum_{k=0}^{n+|m|}\left(\begin{array}{c}
n \\
j
\end{array}\right)\left(\begin{array}{c}
n+|m| \\
k
\end{array}\right) i^{\operatorname{sgn}(m)(k-j)} \\
& \times \sqrt{(2 n+|m|-j-k) !(j+k) !}|2 n+| m|-j-k, j+k\rangle_{\tilde{x}, \tilde{y}}^{c},
\end{aligned}
$$

where $\tilde{r}, \tilde{\phi}$ stands for $R, \phi_{R}$ or $r, \phi_{r}$, and the same convention holds for the Cartesian coordinates, $\operatorname{sgn}(m)=1$ for $m \geqslant 0$ and $\operatorname{sgn}(m)=-1$ for $m<0$. The one-particle Cartesian eigenfunctions corresponding to the vector state $\left|n_{\tilde{x}}, n_{\tilde{y}}\right\rangle^{c}=\left|n_{\tilde{x}}\right\rangle^{c}\left|n_{\tilde{y}}\right|^{c}$ are given by

$$
\left\langle\tilde{x}, \tilde{y} \mid n_{\tilde{x}}, n_{\tilde{y}}\right\rangle^{c}=\sqrt{\frac{\omega_{\tilde{r}}}{\pi 2^{n_{\tilde{x}}+n_{\tilde{y}}+1} n_{\tilde{x}} ! n_{\tilde{y}} !}} e^{-\omega_{\tilde{r}}\left(\tilde{x}^{2}+\tilde{y}^{2}\right) / 4} H_{n_{\tilde{x}}}\left(\sqrt{\frac{\omega_{\tilde{r}}}{2}} \tilde{x}\right) H_{n_{\tilde{y}}}\left(\sqrt{\frac{\omega_{\tilde{r}}}{2}} \tilde{y}\right),
$$

where $H_{n}$ is the Hermite polynomial of $n$th degree, with $n=0,1,2 \ldots$ The energy of these two-dimensional harmonic oscillator states is $E_{n_{\tilde{x}}, n_{\tilde{y}}}=\omega_{\tilde{r}}\left(n_{\tilde{x}}+n_{\tilde{y}}+1\right)$.

Collecting the results above, we get that

$$
|n, m, l, p\rangle=\sum_{j=0}^{n} \sum_{k=0}^{n+|m|} \sum_{r=0}^{l} \sum_{s=0}^{l+|p|} \kappa(n, m, j, k) \kappa(l, p, r, s)|2 n+| m|-j-k\rangle_{x_{1}+x_{2}}^{c}|j+k\rangle_{y_{1}+y_{2}}^{c}|2 l+| p|-r-s\rangle_{x_{1}-x_{2}}^{c}|r+s\rangle_{y_{1}-y_{2}}^{c},
$$


where the notation indicates which oscillator and coordinates must be used to obtain the corresponding eigenfunctions:

$$
\begin{aligned}
\psi_{n, l, m, p}\left(x_{1}, y_{1}, x_{2}, y_{2}\right)= & \sum_{j=0}^{n} \sum_{k=0}^{n+|m|} \sum_{r=0}^{l} \sum_{s=0}^{l+|p|} \kappa(n, m, j, k) \kappa(l, p, r, s) f_{2 n+|m|-j-k}^{\omega_{R}}\left(\frac{x_{1}+x_{2}}{\sqrt{2}}\right) \\
& \times f_{j+k}^{\omega_{R}}\left(\frac{y_{1}+y_{2}}{\sqrt{2}}\right) f_{2 l+|p|-r-s}^{\omega_{r}}\left(\frac{x_{1}-x_{2}}{\sqrt{2}}\right) f_{r+s}^{\omega_{r}}\left(\frac{y_{1}-y_{2}}{\sqrt{2}}\right) \\
= & \sum_{j=0}^{n} \sum_{k=0}^{n+|m|} \sum_{r=0}^{l} \sum_{s=0}^{l+|p|} \kappa(n, m, j, k) \kappa(l, p, r, s) \Upsilon_{2 n+|m|-j-k, j+k, 2 l+|p|-r-s, r+s}\left(x_{1}, y_{1}, x_{2}, y_{2}\right),
\end{aligned}
$$

where $f_{n}^{\omega}(x)$ are the eigenfunctions of a one-dimensional harmonic oscillator of frequency $\omega$ and quantum number $n$ and the last equation defines $\Upsilon_{a, b, c, d}$.

The one-particle reduced density matrix for an eigenfunction $\psi_{n, l, m, p}$ can be obtained exactly from

$$
\rho_{A}\left(\vec{x}_{1}, \vec{x}_{1}^{\prime}\right)=\int d \vec{x}_{2} \psi_{n, m, l, p}^{*}\left(\vec{x}_{1}, \vec{x}_{2}\right) \psi_{n, m, l, p}\left(\vec{x}_{1}^{\prime}, \vec{x}_{2}\right),
$$

where $\psi_{n, m, l, p}\left(\vec{x}_{1}, \vec{x}_{2}\right)$ is the two-particle wave function $|n, m, l, p\rangle$ written in terms of the original particle coordinates, Eq. (B5). Actually, to implement the calculation of the eigenvalues, $\lambda_{i}^{A}$, required to obtain the different entropies that enter into the convexity criterion, Eqs. (12) and (15)-(17), it is useful to calculate the matrix elements of $\psi_{n, m, l, p}$ in a one-particle one-coordinate basis function $\phi_{i}(z)$, where $z$ stands for $x$ or $y$, i.e., we first calculate

$$
K_{i_{1}, j_{1}, i_{2}, j_{2}}^{n, m, l}=\int d x_{1} d y_{1} d x_{1}^{\prime} d y_{1}^{\prime} \phi_{i_{1}}\left(x_{1}\right) \phi_{j_{1}}\left(y_{1}\right) \psi_{n, m, l, p}\left(x_{1}, y_{1}, x_{1}^{\prime}, y_{1}^{\prime}\right) \phi_{i_{2}}\left(x_{1}^{\prime}\right) \phi_{j_{2}}\left(y_{1}^{\prime}\right)
$$

and then solve the eigenvalue problem

$$
\mathbf{K} \mathbf{u}_{i}=k_{i}^{A} \mathbf{u}
$$

where $\mathbf{K}$ is the matrix the entries of which are given by Eq. (B7). Since $\psi_{n, m, l, p}$ is a symmetric kernel (under particle exchange), the eigenvalues of the reduced density matrix satisfy that [41]

$$
\lambda_{i}^{A}=\left(k_{i}^{A}\right)^{2} .
$$

The algebra involved in the calculation of the elements $K_{i_{1}, j_{1}, i_{2}, j_{2}}^{n, m, l, p}$ is rather cumbersome, but direct, so we write them explicitly. We compute the expressions for the wave function of two noninteracting harmonic oscillators, $\lambda=0$, and also the elements of its reduced density matrix, Eq. (B7). The corresponding quantities for $\lambda \neq 0$ can be obtained following a completely equivalent procedure.

Then, using the expansion of the Hermite polynomials [42], we obtain

$$
\begin{aligned}
\Upsilon_{n, m, l, p}\left(x_{1}, y_{1}, x_{2}, y_{2}\right)= & \sqrt{\frac{n ! m ! l ! p !}{\pi^{2} 2^{n+m+l+p}} \sum_{a=0}^{\lfloor n / 2\rfloor} \sum_{b=0}^{\lfloor m / 2\rfloor} \sum_{c=0}^{\lfloor l / 2\rfloor} \sum_{d=0}^{\lfloor p / 2\rfloor} \sum_{s=0}^{n-2 a} \sum_{t=0}^{m-2 b} \sum_{v=0}^{l-2 c} \sum_{w=0}^{p-2 d}\left(\begin{array}{c}
n-2 a \\
s
\end{array}\right)\left(\begin{array}{c}
m-2 b \\
t
\end{array}\right)} \\
& \times\left(\begin{array}{c}
l-2 c \\
v
\end{array}\right)\left(\begin{array}{c}
p-2 d \\
w
\end{array}\right) \frac{(-1)^{a+b+c+d+v+w} \sqrt{2}{ }^{n-2 a+m-2 b+l-2 c+p-2 d}}{a ! b ! c ! d !(n-2 a) !(m-2 b) !(l-2 c) !(p-2 d) !} \\
& \times x_{2}^{n-2 a-s+l-2 c-v} y_{1}^{m-2 b-t+p-2 d-w} x_{1}^{s+v} y_{2}^{t+w} e^{-\left(x_{1}^{2}+y_{1}^{2}+x_{2}^{2}+y_{2}^{2}\right) / 2} .
\end{aligned}
$$

Now, for each $\Upsilon$ function we construct a kernel $K_{\Upsilon}$ by means of Eq. (B7), and using the one-particle basis functions $\phi_{k}=f_{k}^{1}(x)$ we obtain the matrix representation of each kernel as

$$
\begin{aligned}
{\left[K_{\left.\Upsilon_{n, m, l, p}\right]} i_{i_{1}, j_{1}, i_{2}, j_{2}}=\right.} & \sqrt{\frac{n ! m ! l ! p ! i_{1} ! j_{1} ! i_{2} ! j_{2} !}{\pi^{4} 2^{n+m+l+p+i_{1}+j_{1}+i_{2}+j_{2}}} \sum_{a=0}^{\lfloor n / 2\rfloor} \sum_{b=0}^{\lfloor m / 2\rfloor} \sum_{c=0}^{\lfloor l / 2\rfloor} \sum_{d=0}^{\lfloor p / 2\rfloor} \sum_{s=0}^{n-2 a} \sum_{t=0}^{m-2 b} \sum_{v=0}^{l-2 c} \sum_{w=0}^{p-2 d}} \\
& \times \sum_{q=0}^{\left\lfloor i_{1} / 2\right\rfloor} \sum_{z=0}^{\left\lfloor j_{1} / 2\right\rfloor} \sum_{g=0}^{\left\lfloor i_{2} / 2\right\rfloor} \sum_{e=0}^{\left\lfloor j_{2} / 2\right\rfloor}\left(\begin{array}{c}
n-2 a \\
s
\end{array}\right)\left(\begin{array}{c}
m-2 b \\
t
\end{array}\right)\left(\begin{array}{c}
l-2 c \\
v
\end{array}\right)\left(\begin{array}{c}
p-2 d \\
w
\end{array}\right) \\
& \times \frac{(-1)^{a+b+c+d+v+w+q+z+g+e} \sqrt{2}^{n-2 a+m-2 b+l-2 c+p-2 d} 2^{i_{1}-2 q+j_{1}-2 z+i_{2}-2 g+j_{2}-2 e}}{a ! b ! c ! d !(n-2 a) !(m-2 b) !(l-2 c) !(p-2 d) !\left(i_{1}-2 q\right) !\left(j_{1}-2 z\right) !\left(i_{2}-2 g\right) !\left(j_{2}-2 e\right) !}
\end{aligned}
$$




$$
\begin{aligned}
& \times \int_{-\infty}^{\infty} x_{1}^{n-2 a-s+l-2 c-v+i_{1}-2 q} e^{-x_{1}^{2}} d x_{1} \int_{-\infty}^{\infty} y_{1}^{m-2 b-t+p-2 d-w+j_{1}-2 z} e^{-y_{1}^{2}} d y_{1} \\
& \times \int_{-\infty}^{\infty} x_{2}^{s+v+i_{2}-2 g} e^{-x_{2}^{2}} d x_{2} \int_{-\infty}^{\infty} y_{2}^{t+w+j_{2}-2 e} e^{-y_{2}^{2}} d y_{2} .
\end{aligned}
$$

All the integrals that appear in the last expression are obtained in terms of the Gamma function [43]. Finally, the matrix representation of $\mathbf{K}$ in Eq. (B8) can be written as

$$
[\mathbf{K}]_{i_{1}, j_{1}, i_{2}, j_{2}}=\sum_{j=0}^{n} \sum_{k=0}^{n+|m|} \sum_{r=0}^{l} \sum_{s=0}^{l+|p|} \kappa(n, m, j, k) \kappa(l, p, r, s)\left[K_{\Upsilon_{2 n+|m|-j-k, j+k, 2 l+|p|-r-s, r+s}}\right]_{i_{1}, j_{1}, i_{2}, j_{2}} .
$$

[1] N. Gershenfeld and I. L. Chuang, Science 275, 350 (1997).

[2] D. G. Cory, A. F. Fahmy, and T. F. Havel, Proc. Natl. Acad. Sci. USA 94, 1634 (1997).

[3] L. M. K. Vandersypen, M. Steffen, G. Breyta, C. S. Yannoni, M. H. Sherwood, and I. L. Chuang, Nature (London) 414, 883 (2001).

[4] J. R. Petta, A. C. Johnson, J. M. Taylor, E. A. Laird, A. Yacoby, M. D. Lukin, C. M. Marcus, M. P. Hanson, and A. C. Gossard, Science 309, 2180 (2005).

[5] M. Hofheinz, H. Wang, M. Ansmann, R. C. Bialczak, E. Lucero, M. Neeley, A. D. O'Connell, D. Sank, J. Wenner, J. M. Martinis, and A. N. Cleland, Nature (London) 459, 546 (2009).

[6] E. Knill, R. Laflamme, and G. J. Milburn, Nature (London) 409, 46 (2001).

[7] D. S. Abrams and S. Lloyd, Phys. Rev. Lett. 83, 5162 (1999).

[8] J. L. O'Brien, A. Furusawa, and J. Vučković, Nat. Photonics 3, 687 (2009).

[9] R. Lo Franco and G. Compagno, Phys. Rev. Lett. 120, 240403 (2018).

[10] A. Vaziri, G. Weihs, and A. Zeilinger, J. Opt. B 4, S47 (2002).

[11] B. Jack, J. Leach, H. Ritsch, S. M. Barnett, M. J. Padgett, and S. Franke-Arnold, New J. Phys. 11, 103024 (2009).

[12] G. Parisi, E. Mari, F. Spinello, F. Romanato, and F. Tamburini, Opt. Express 22, 17135 (2014).

[13] V. V. Kotlyar, A. A. Kovalev, and A. P. Porfirev, Phys. Rev. A 95, 053805 (2017).

[14] F. Schlederer, M. Krenn, R. Fickler, M. Malik, and A. Zeilinger, New J. Phys. 18, 043019 (2016).

[15] R. Dall, Michael D. Fraser, A. S. Desyatnikov, G. Li, S. Brodbeck, M. Kamp, C. Schneider, S. Höfling, and E. A. Ostrovskaya, Phys. Rev. Lett. 113, 200404 (2014).

[16] T. J. Osborne and M. A. Nielsen, Phys. Rev. A 66, 032110 (2002).

[17] M. Gaudiano, O. Osenda, and G. A. Raggio, Phys. Rev. A 77, 022109 (2008).

[18] D. J. H. Markham, Phys. Rev. A 83, 042332 (2011).

[19] A. Hamma, S. M. Giampaolo, and F. Illuminati, Phys. Rev. A 93, 012303 (2016).

[20] L. Vidmar, L. Hackl, E. Bianchi, and M. Rigol, Phys. Rev. Lett. 119, 020601 (2017).

[21] L. Vidmar and M. Rigol, Phys. Rev. Lett. 119, 220603 (2017).

[22] M. Garagiola, E. Cuestas, F. M. Pont, P. Serra, and O. Osenda, Phys. Rev. A 94, 042115 (2016).

[23] B. D. Simons, P. A. Lee, and B. L. Altshuler, Phys. Rev. Lett. 70, 4122 (1993); B. D. Simons and B. L. Altshuler, Phys. Rev. B 48, 5422 (1993); H. Azuma and S. Iso, Phys. Lett. B 331, 107
(1994); M. V. N. Murthy and R. Shankar, Phys. Rev. Lett. 73, 3331 (1994); A. P. Polychronakos, J. Phys. A 39, 12793 (2006).

[24] P.-F. Loos and P. M. W. Gill, Phys. Rev. Lett. 103, 123008 (2009).

[25] I. V. Toranzo, A. R. Plastino, P. Sánchez-Moreno, and J. S. Dehesa, J. Phys. A 48, 475302 (2015).

[26] P. Kok and B. W. Lovett, Introduction to Optical Quantum Information Processing (Cambridge University, Cambridge, England, 2010).

[27] M. Garagiola, F. M. Pont, and O. Osenda, J. Phys. B 51, 075504 (2018).

[28] M. Ali Can, A. Klyachko, and A. Shumovsky, J. Opt. B 7, L1 (2005).

[29] J. S. Dehesa, I. V. Toranzo, and D. Puertas-Centeno, Int. J. Quantum Chem. 117, 48 (2017).

[30] M. A. Nielsen and I. L. Chuang, Quantum Computation and Quantum Information (Cambridge University, Cambridge, England, 2000).

[31] C. Cohen-Tannoudji, B. Diu, and F. Laloe, Quantum Mechanics (Wiley, New York, 1977).

[32] C.-H. Lin and Y. K. Ho, Few-Body Syst. 56, 157 (2015).

[33] C. A. Downing, Phys. Rev. A 95, 022105 (2017); V. Tognetti and P.-F. Loos, J. Chem. Phys. 144, 054108 (2016); C. A. Downing, J. Math. Phys. 54, 072101 (2013); C. A. Downing and M. E. Portnoi, Phys. Rev. B 94, 045430 (2016).

[34] F. M. Pont, O. Osenda, and P. Serra, J. Phys. A 51, 195303 (2018).

[35] G. S. Ezra and R. S. Berry, Phys. Rev. A 25, 1513 (1982) ; 28, 1989 (1983); P. C. Ojha and R. S. Berry, ibid. 36, 1575 (1987); R. J. Hinde and R. S. Berry, ibid. 42, 2259 (1990); J. W. Warner and R. S. Berry, Nature (London) 313, 160 (1985).

[36] C. Schwartz, Phys. Rev. 123, 1700 (1961); H. F. King, J. Chem. Phys. 46, 705 (1967); C. Bottcher, D. R. Schultz, and D. H. Madison, Phys. Rev. A 49, 1714 (1994).

[37] G. Pestka, J. Phys. A 41, 235202 (2008).

[38] J. F. Perkins, J. Chem. Phys. 48, 1985 (1968).

[39] S. Gigan, L. Lopez, N. Treps, A. Maître, and C. Fabre, Phys. Rev. A 72, 023804 (2005).

[40] Z.-D. Cheng et al., Opt. Lett. 42, 2042 (2017).

[41] K. J. H. Giesbertz and R. van Leeuwen, J. Chem. Phys. 139, 104109 (2013); F. G. Tricomi, Integral Equations (Interscience, New York, 1957).

[42] I. S. Gradshteyn and I. M. Ryzhik, Table of Integrals, Series and Products, 7th ed. (Elsevier, New York, 2007).

[43] M. Abramowitz and I. A. Stegun, Handbook of Mathematical Functions with Formulas, Graphs, and Mathematical Tables, 9th ed. (Dover, New York, 1970). 